\title{
Martian Moons Exploration MMX: Sample Return Mission to Phobos Elucidating Formation Processes of Habitable Planets
}

Kiyoshi Kuramoto ( $\square$ keikei@ep.sci.hokudai.ac.jp)

Hokkaido University https://orcid.org/0000-0002-6757-8064

Yasuhiro Kawakatsu

JAXA

Masaki Fujimoto

JAXA

Akito Araya

The University of Tokyo: Tokyo Daigaku

Maria Antonietta Barucci

Paris Observatory: Observatoire de Paris

Hidenori Genda

Tokyo Institute of Technology

Naru Hirata

The University of Aizu: Aizu Daigaku

Hitoshi lkeda

JAXA

Takeshi Imamura

The University of Tokyo: Tokyo Daigaku

Jörn Helbert

Deutsches Zentrum fur Luft- und Raumfahrt DLR

Shingo Kameda

Rikkyo University

Masanori Kobayashi

Chiba Institute of Technology

Hiroki Kusano

National Institutes for Quantum and Radiological Science and Technology

David J. Lawrence

Johns Hopkins University Applied Physics Laboratory

Koji Matsumoto

National Astronomical Observatory of Japan

Patrick Michel 
Universite Cote d'Azur

\section{Hideaki Miyamoto}

The University of Tokyo: Tokyo Daigaku

\section{Tomokatsu Morota}

The University of Tokyo: Tokyo Daigaku

\section{Hiromu Nakagawa}

Tohoku University: Tohoku Daigaku

\section{Tomoki Nakamura}

Tohoku University

\section{Kazunori Ogawa}

JAXA

Hisashi Otake

JAXA

Masanobu Ozaki

JAXA

\section{Sara Russel}

Natural History Museum

\section{Sho Sasaki}

Osaka University: Osaka Daigaku

\section{Hirotaka Sawada}

JAXA

\section{Hiroki Senshu}

Chiba Institute of Technology: Chiba Kogyo Daigaku

\section{Shogo Tachibana}

The University of Tokyo: Tokyo Daigaku

\section{Naoki Terada}

Tohoku University: Tohoku Daigaku

\section{Stephan Ulamec}

Deutsches Zentrum für Luft- und Raumfahrt DRL

\section{Tomohiro Usui}

JAXA

\section{Koji Wada}

Chiba Institute of Technology: Chiba Kogyo Daigaku

\section{Sei-ichiro Watababe}

Nagoya University: Nagoya Daigaku

\section{Shoichiro Yokota}

Osaka University: Osaka Daigaku 
Frontier Letter (by invitation only)

Keywords: Phobos, Deimos, Mars, sample return mission, early solar system, habitable planet

Posted Date: February 3rd, 2021

DOl: https://doi.org/10.21203/rs.3.rs-163944/v1

License: (c) (i) This work is licensed under a Creative Commons Attribution 4.0 International License. Read Full License

Version of Record: A version of this preprint was published at Earth, Planets and Space on January 20th, 2022. See the published version at https://doi.org/10.1186/s40623-021-01545-7. 


\section{Title: Martian Moons Exploration MMX: Sample Return}

\section{Mission to Phobos Elucidating Formation Processes of}

\section{$3 \quad$ Habitable Planets}

4 Author \#1: *Kiyoshi Kuramoto, Hokkaido University, Sapporo, Japan/Institute of Space

5 and Astronautical Science, Japan Aerospace Exploration Agency, Sagamihara, Japan,

$6 \quad$ keikei@ep.sci.hokudai.ac.jp

7 Author \#2: Yasuhiro Kawakatsu Institute of Space and Astronautical Science, Japan

8 Aerospace Exploration Agency, Sagamihara, Japan, kawakatsu.yasuhiro@jaxa.jp

9 Author \#3: Masaki Fujimoto, Institute of Space and Astronautical Science, Japan

10 Aerospace Exploration Agency, Sagamihara, Japan, fujimoto.masaki@jaxa.jp

11 Author \#4: Akito Araya, The University of Tokyo, Tokyo, Japan, araya@eri.u-

12 tokyo.ac.jp

13 Author \#5: Maria Antonietta Barucci, Laboratoire d'Etudes Spatiales et d'Instrumentation en

14 Astrophysique, Paris Observatory, Meudon, France, antonella.barucci@obspm.fr

15 Author \#6: Hidenori Genda, Tokyo Institute of Technology, Tokyo, Japan,

16 genda@elsi.jp 
17 Author \#7: Naru Hirata, Aizu University, Aizu Wakamatsu, Japan, naru@u-aizu.ac.jp

18 Author \#8: Hitoshi Ikeda, Institute of Space and Astronautical Science, Japan Aerospace

19 Exploration Agency, Sagamihara, Japan, ikeda.hitoshi@jaxa.jp

20 Author \#9: Takeshi Imamura, The University of Tokyo, Tokyo, Japan,

21 t_imamura@edu.k.u-tokyo.ac.jp

22 Author \#10: Jörn Helbert, Deutsches Zentrums für Luft- und Raumfahrt, Berlin,

23 Germany, Joern.Helbert@dlr.de

24 Author \#11: Shingo Kameda, Rikkyo University, Tokyo, Japan, kameda@rikkyo.ac.jp

25 Author \#12: Masanori Kobayashi, Chiba Institute of Technology, Narashino, Japan,

26 kobayashi.masanori@it-chiba.ac.jp

27 Author \#13: Hiroki Kusano, National Institutes for Quantum and Radiological Science

28 and Technology, Chiba, Japan, kusano.hiroki@qst.go.jp

29 Author \#14: David J. Lawrence, Johns Hopkins University Applied Physics Laboratory,

30 Laure, USA, David.J.Lawrence@jhuapl.edu

31 Author \#15: Koji Matsumoto, National Astronomical Observatory of Japan, Mitaka,

32 Japan, koji.matsumoto@nao.ac.jp 
33 Author \#16: Patrick Michel, Université Côte d'azur, Observatoire de la Côte d'Azur,

34 CNRS, Laboratoire Lagrange, Nice, France, michelp@oca.eu

35 Author \#17: Hideaki Miyamoto, The University of Tokyo, Tokyo, Japan, hm@sys.t.u-

36 tokyo.ac.jp

37 Author \#18: Tomokatsu Morota, The University of Tokyo, Tokyo, Japan,

38 morota@eps.s.u-tokyo.ac.jp

39 Author \#19: Hiromu Nakagawa, Tohoku University, Sendai, Japan,

40 hiromu.nakagawa.c1@tohoku.ac.jp

41 Author \#20: Tomoki Nakamura, Tohoku University, Sendai, Japan,

42 tomoki.nakamura.a8@tohoku.ac.jp

43 Author \#21: Kazunori Ogawa, Institute of Space and Astronautical Science, Japan

44 Aerospace Exploration Agency, Sagamihara, Japan, ogawa.kazunori@jaxa.jp

45 Author \#22: Hisashi Otake, Institute of Space and Astronautical Science, Japan

46 Aerospace Exploration Agency, Sagamihara, Japan, ootake.hisashi@jaxa.jp

47 Author \#23: Masanobu Ozaki, Institute of Space and Astronautical Science, Japan

48 Aerospace Exploration Agency, Sagamihara, Japan, ozaki.masanobu@jaxa.jp 
49 Author \#24: Sara Russel, Natural History Museum, London, UK,

50 sara.russell@nhm.ac.uk

51 Author \#25: Sho Sasaki, Osaka University, Osaka, Japan, sasakisho@ess.sci.osaka-

$52 \quad$ u.ac.jp

53 Author \#26: Hirotaka Sawada, Institute of Space and Astronautical Science, Japan

54 Aerospace Exploration Agency, Sagamihara, Japan, sawada.hirotaka@jaxa.jp

55 Author \#27: Hiroki Senshu, Chiba Institute of Technology, Narashino, Japan,

56 senshu@perc.it-chiba.ac.jp

57 Author \#28: Shogo Tachibana, The University of Tokyo, Tokyo, Japan /Institute of

58 Space and Astronautical Science, Japan Aerospace Exploration Agency, Sagamihara,

$59 \quad$ Japan, tachi@eps.s.u-tokyo.ac.jp

60 Author \#29: Naoki Terada, Tohoku University, Sendai, Japan,

61 teradan@pat.gp.tohoku.ac.jp

62 Author \#30: Stephan Ulamec, Deutsches Zentrums für Luft- und Raumfahrt, Berlin,

63 Germany, Stephan.Ulamec@dlr.de

64 Author \#31: Tomohiro Usui, Institute of Space and Astronautical Science, Japan 
65 Aerospace Exploration Agency, Sagamihara, Japan, usui.tomohiro@jaxa.jp

66 Author \#32: Koji Wada, Chiba Institute of Technology, Narashino, Japan,

67 wada@perc.it-chiba.ac.jp

68 Author \#33: Sei-ichiro Watababe, Nagoya University, Nagoya, Japan,

69 seicoro@eps.nagoya-u.ac.jp

70 Author \#34: Shoichiro Yokota, Osaka University, Osaka, Japan, yokota@ess.sci.osaka-

$71 \quad$ u.ac.jp

72

73 *The corresponding author

74 


\section{Abstract}

76 Martian moons exploration, MMX, is the new sample return mission planned by the

77 Japan Aerospace Exploration Agency (JAXA) targeting the two Martian moons with a

78 scheduled launch in 2024 and a return to the Earth in 2029. The major scientific

79 objectives of this mission are to determine the origin of Phobos and Deimos, to

80 elucidate the early Solar System evolution in terms of volatile delivery across the snow

81 line to the terrestrial planets having habitable surface environments, and to explore the

82 evolutionary processes of both moons and Mars surface environment. To achieve these

83 objectives, during a stay in circum-Martian space over about 3 years MMX will collect

84 samples from Phobos along with close-up observations of this inner moon and carry out

85 multiple flybys of Deimos to make comparative observations of this outer moon.

86 Simultaneously, successive observations of the Martian atmosphere will also be made

87 by utilizing the advantage of quasi-equatorial spacecraft orbits along the moons' orbits.

89 Keywords

90 Phobos, Deimos, Mars, sample return mission, early solar system, habitable planet 


\section{$91 \quad$ Introduction}

93 Amongst the numerous moons currently known in the Solar System, there are only three

94 orbiting terrestrial planets, Phobos and Deimos being the two of them. The exploration

95 of the Earth's Moon, which includes sample return missions, has provided us a wealth

96 of data revealing and constraining both its formation and evolution processes and those

97 of the parent planet. The same is probably true for the two Martian moons which also

98 likely formed associated with the formation of their parent planet. However, the direct

99 exploration of the Martian moons has been quite limited so far.

100

101 The Martian Moons eXploration (MMX) mission will carry out a direct, extensive

102 survey of the Martian moons including the first sample return from one of these moons.

103 The past explorations of the Martian moons were largely limited to flybys by orbiters

104 approaching Mars. The first close-up imaging of Phobos and Deimos was conducted by

105 the Viking mission, which found their irregular shapes and their albedos as low as those

106 of asteroids thought to have carbonaceous compositions (Veverka and Duxbury 1977;

107 Tolson et al. 1978; Pang et al. 1978; Pang et al. 1980). This led to the asteroid capture 
108 hypothesis for the origin of both moons (Pollack et al 1979; Hunten 1979). Direct

109 exploration of Phobos was attempted by Phobos 1 (1988), Phobos 2 (1988-1989), and

110 Phobos-Grunt (2011), but they, unfortunately, failed except for valuable, but incomplete

111 data acquisition by Phobos 2 in the vicinity of Phobos (Duxbury et al. 2014 for a

112 comprehensive summary of the past explorations of Phobos and Deimos). Although

113 several mission concepts have been proposed to explore the Martian moons, MMX is

114 currently the only approved mission targeting the Martian moons.

115

116 For the last two decades, JAXA has been a pioneer in small body sample return

117 missions, accumulating unique experience through the first asteroid sample return

118 mission Hayabusa (originally named MUSES-C, 2003-2010), to the S-type asteroid

119 Itokawa followed by the Hayabusa2 mission (2014-present) to the C-type asteroid

120 Ryugu. Both missions have been successfully revealing how solid materials were built

121 up to form small bodies which then migrated to near-Earth orbits involving disruption

122 and re-accumulation processes during Solar System evolution (e.g. Fujiwara et al. 2006;

123 Watanabe et al. 2019; Sugita et al. 2019; Michel et al. 2020). In particular, the

124 Hayabusa2 mission is elucidating processes that may have supplied water and organics 
to Earth. MMX will expand these Japanese experiences and successes in small body

explorations to the Martian moons.

127

128 As a Mars orbiter, on the other hand, MMX will extend JAXA's experiences in

129 gravitational body explorations gained by Kaguya (SELENE, 2007-2009) at the Moon

130 and ongoing Akatsuki (Planet-C, 2010-present) at Venus. The attempted Japanese Mars

131 orbiter Nozomi (Planet-B, 1998-2003) launched by ISAS before the institution

132 integration into JAXA, unfortunately, resulted in contact loss during the cruise to Mars.

133 Hence, the MMX mission is an important milestone to expand the Japanese space

134 program to Mars synergistically with small body exploration programs.

135

136 This paper presents the current design of the MMX mission with a special focus on the

137 scientific objectives as well as the mission requirements, the system architecture, and

138 the observation operations plan to achieve those objectives. Note that the MMX mission

139 is under development therefore its details may be modified in the future.

140

141 Phobos, Deimos, and Mars: Genetic and Evolutionary Links 142

143 Mars, the host planet of Phobos and Deimos, is the planet that has an atmosphere- 
144 covered surface environment most analogous to that of the Earth. Abundant fluvial

145 geomorphological features such as valley networks and outflow channels strongly imply

146 the activities of a vast amount of liquid water on the Martian surface before $~ 3$ Gya

147 (e.g. Carr 1996). Long-term volcanic activities leaving igneous provinces and volcanoes

148 may significantly contribute to volatile supply from the interior to the surface (e.g.

149 Greely, 1987). Recent precise imaging spectroscopy from orbits (e.g. Bibring et al.

150 2006) and in-situ geological surveys conducted by rovers (e.g. Grotzinger et al. 2014)

151 accumulate evidence for the widespread existence and past activities of liquid water

152 with compositions suitable for habitats. On the other hand, Mars preserves ancient

153 terrains with numerous impact craters and may be the only planet for which we can

154 precisely trace the history back to the early evolution of a hydrosphere on a rocky

155 planet. It seems, however, difficult to approach Mars formation processes through Mars

156 surface exploration alone because resurfacing processes significantly obscure records of

157 the planet formation. In contrast, the airless Martian moons with sizes too small to

158 activate long-term igneous processes likely preserve materials at their formation without

159 severe alteration, possibly providing a window to explore key processes forming Mars. 
161 The size of Mars, which is about half of that of Earth, and the formation time of Mars

162 which is within several Myr since the start of the Solar System constrained from

163 radioisotope studies (Dauphas and Pourmand 2011) are consistent with the typical

164 characteristics of proto-planets predicted by planet formation theory (Kokubo and Ida

165 1998). Here, a proto-planet refers to the product of oligarchic growth that would have

166 occurred in a localized feeding zone of the planetesimal population. As a result of

167 oligarchic growth, the inner Solar System may have once contained dozens of proto-

168 planets with typical masses from lunar to Martian ones. Their subsequent mutual

169 collisions that occurred episodically over the time scale several $10^{7}$ years mostly after

170 the dissipation of solar nebula gas would lead the proto-Earth and proto-Venus to

171 accumulating their current masses (Kokubo and Ida 1998). In contrast, isotopic

172 heterogeneities of extinct radionuclide systematics observed in Martian meteorites

173 suggest insufficient mantle mixing of Mars, implying the lack of complete melting

174 event possibly induced by the collision of a proto-planet (Debaille et al. 2007; Kleine et

175 al. 2009). As a fossil of proto-planet, therefore, Mars may be the unique research target

176 for revealing the processes of proto-planet formation and evolution. Inhibition of the 
177 growth of Mars exceeding the present mass implies that the population of planetesimals

178 in the early Solar System rapidly declined in the region beyond the current orbital radius

179 of Mars, which might be caused by the gravitational perturbation of proto-Jupiter that

180 once migrated inward significantly (Walsh et al. 2011). This proposed mechanism may

181 have also played an important role in delivering water-bearing objects formed in the

182 outer Solar System to the terrestrial planets. The exploration of Martian moons may

183 place important constraints on such a model.

184

185 The presence of water and atmosphere is believed to be a primarily necessary condition

186 to make a rocky planet habitable. Although the origin of water and other volatiles on the

187 terrestrial planets remains controversial, one of the dominant hypotheses is the delivery

188 by late accreting bodies originated in the outer Solar System (Genda 2016 for review).

189 This is because the rocky planets are thought to be born dry if they were made from

190 solid materials accreted in the inner solar nebula where nebular gas was too warm for

191 water vapor to condense onto dust. In the outer solar nebula, beyond the snow line,

192 water may condense as ice, which allows the formation of icy planetesimals. A part of

193 them may further evolve and migrate to become rocky asteroids in the asteroid belt (e.g. 
194 Walsh et al. 2011), containing hydrated minerals generated through the chemical

195 reactions of silicates with liquid water produced by internal heating (e.g. Fujiya et al.

196 2012). Observed compositions of meteorites and comets suggest that such hydrated and

197 icy bodies are also enriched in carbon and nitrogen mainly in the form of complex

198 organic matter (e.g. Kallemeyn and Wasson 1981; Mumma and Charnley 2011). Mars is

199 in the best position to elucidate how such volatile-rich bodies are transported in the

200 early Solar System because it is the terrestrial planet orbiting nearest the snow line.

201

202 Compared to the Earth's Moon, Phobos and Deimos are characterized by their smallness

203 in size, low bulk densities, irregular shapes, and very low albedos (Table 1). They share

204 visible to near-infrared reflectance spectra with reddening trends like those of D-type

205 and T-type asteroids which are thought to have volatile-rich, carbonaceous compositions

206 (Rivkin et al. 2002). Their small sizes and low bulk densities appear to be within the

207 range of undifferentiated small bodies (e.g. Burns 1978). According to the spectroscopic

208 classification of asteroids, the above types of asteroids are likely originated in the region

209 near the Jovian orbit (DeMeo and Carry 2014). These facts naturally lead to the capture

210 hypothesis for the origin of the Martian moons; carbonaceous primitive bodies 
211 originally formed beyond the snow line of the Solar System might have migrated

212 inward (Walsh et al. 2011) and then become captured by Mars. If this is the case, the

213 exploration of Martian moons will provide us clues for the delivery processes of water

214 and other volatiles from the outer Solar System to early terrestrial planets.

215 (Table 1)

216

217 The feasibility of asteroid capture to form the Martian moons is, however, controversial.

218 To capture a heliocentric body as a satellite, dissipation of orbital energy relative to the

219 planet is required. Models of aerodynamic capture have suggested that heliocentric

220 bodies with sizes similar to Phobos and Deimos are possibly captured by proto-Mars

221 embedded in the solar nebula gas (Hunten 1979; Pollack et al. 1979). The orbit of a

222 captured body gradually circularizes and then decays toward a collision with the

223 planetary surface. Thus, the aerodynamic capture scenario requires some mechanism to

224 terminate the action of aerodynamic drag, such as the loss of an extended atmosphere

225 containing a small number of capture bodies (Hunten 1979). On the other hand, Phobos

226 and Deimos have low orbital inclinations and eccentricities (Table 1). Since heliocentric 
227 bodies that encounter a planet may approach from almost random directions, the

228 resulting orbital inclinations of captured bodies are likely also random relative to the

229 planetary equatorial plane as seen in irregular satellites of giant planets. Tidal

230 interactions with Mars seem too weak to reduce the orbital inclinations of captured

231 bodies during its history given initial large inclinations (Rosenblatt 2011). An

232 alternative mechanism to reduce inclination may be gas-drag in a proto-atmosphere or

233 circum-planetary gas envelope rotated in the same direction of the planetary rotation. It

234 remains, however, poorly understood how such atmospheric conditions may be

235 established and effectively work to produce moons with near-equatorial orbits.

236

237 The giant impact origin hypothesis, the other leading theory for the formation of the

238 Martian moons, could satisfy the constraints from their orbits. Tidal interactions with

239 Mars make Phobos orbit decline and Deimos one expand. Hence, their original orbits

240 would be near the co-rotation radius of Mars located at a distance of $\sim 6$ Martian radii

241 from the planetary center (Burns 1978). Recent dynamical simulations of impact ejecta

242 released from huge (potential) impact structures with a scale comparable to the Borealis 
243 basin, for example, succeed to explain small masses and near-circular equatorial orbits

244 of both moons (Rosenblatt et al. 2016; Canup and Salmon 2018). To reconcile with the

245 formation of moons near the co-rotation radius, the total mass of impact ejecta extended

246 to several Martian radii should be much larger than that of both moons, thereby leading

247 to the formation of an inner large moon through the accretion of ejecta materials. Such a

248 large moon may gravitationally excite the random motion of debris in the outer ejecta

249 disk and promote their collisional coagulation into a small number of tiny moons. The

250 inner large moon would eventually fall onto Mars due to tidal interactions leaving the

251 two tiny moons with near-circular and near-equatorial orbits in the vicinity of the co-

252 rotation radius of Mars. In an extended giant impact origin scenario, Phobos may be the

253 youngest generation of the inner moon that has repeated tidal break-ups, followed by

254 orbital diffusion of disrupted debris and their partial re-accumulation during the secular

255 tidal orbital evolution (Hesselbrock and Minton, 2017). Note, however, that clear

256 geologic evidence has been yet unidentified for the mass loss of large inner moon onto

257 the Martian surface so far. 
259 An elaborate numerical analysis of the giant impact model for the origin of the Martian

260 moons predicts that the Martian moons consist of a near 50:50 mixture of impactor

261 materials and proto-Mars materials (Hyodo et al. 2017). If this is the case, the Martian

262 moons will provide us clues for the composition of both the impactor and the proto-

263 Mars, which will also allow improving drastically our understanding of the giant impact

264 phenomena that resulted in the formation of both moons. For instance, high energy

265 density gained from the giant impact process likely left igneous mineralogy and texture

266 for the Martian moon materials. On the other hand, it remains unclear how such

267 materials lead to the current reflectance spectra of Phobos and Deimos.

268

269 Altogether, the origin of the Martian moons is still controversial to date. The current

270 pros and cons of the two major hypotheses of the Martian moon origin are summarized

271 in Table 2. Of course, one cannot rule out alternative scenarios such as the co-accretion

272 of moons around a growing Mars. If MMX results lead to an alternative scenario for the

273 origin of Martian moons, unexpected new aspects will be revealed not only for both

274 moons but also for the formation of Mars.

275 (Table2) 
277 Phobos, Deimos, and their surrounding space are also expected to contain clues to

278 understand the evolutionary processes not only on both moons but also on Mars, such as

279 impact flux to the Mars-moons system, resurfacing of satellite surfaces by meteoroid

280 impacts, contamination of impact ejecta from Mars onto the moons' surface, the

281 outflow of the Martian atmosphere, implantation of particles originated from solar wind

282 and Martian atmosphere, possible gas emission from the interiors of the moons, possible

283 formation of dust ring or torus along the moons orbits, and tidal deformation. These are

284 valuable processes to be explored by missions to the Martian moons. Also, from the

285 space around the near-equatorial orbits of the Martian moons, a spacecraft can make

286 monitoring observations of the Martian atmosphere by taking successive images and

287 spectra covering the wide-area of the planet's hemisphere. Such observations are

288 complementary to the low altitude, close-up observations conducted by the previous

289 Mars orbiters.

290

291 Mission Objectives and Requirements 


\section{Mission objectives}

293 To reveal the origin of the Martian moons and elucidate the evolution of Mars and the

294 habitable terrestrial planets in the Solar System, the MMX mission has selected Phobos

295 as the target for sampling and thus for detailed observations. Of course, it would be

296 ideal to take samples from both moons, but this is infeasible because of the limitation of

297 mission resources. The reasons for choosing Phobos as the sampling target are as

298 follows: First, the surface of Phobos exhibits a larger spectral diversity than Deimos

299 (Rivkin et al. 2002; Fraeman et al. 2014). This implies that the composition of Deimos

300 may be within the range of compositional diversity of Phobos material. Note that

301 spectral diversity might also reflect differences in physical states such as grain size

302 distribution of surface regolith rather than compositional difference. Second, Phobos'

303 surface likely contains a certain amount of materials ejected from young impact craters

304 on Mars (Ramsley and Head 2013; Hyodo et al. 2019), which might provide us

305 information on the past surface environment of Mars at the time of bedrock formation

306 before cratering (Usui et al. 2019). Third, more abundant imaging data have been

307 accumulated for Phobos, which allows us to optimize the landing strategies and 
operations before the launch of the spacecraft.

309

310 Referring to the advantage offered by the Martian moons for elucidating processes

311 producing habitable terrestrial planets, the MMX mission places scientific objectives as

312 listed in Table 3, where the major goals and medium objectives flow down to the

313 specific objectives and mission requirements. The two major goals are to elucidate the

314 origin of terrestrial planets with habitable environments and to identify and characterize

315 important evolutionary processes of the Mars system consisting of Mars, its moons, and

316 the circum-Martian space. As for the former goal, the MMX mission aims first to reveal

317 the origin of the Martian moons (medium objective 1.1). Either of the medium

318 objectives $1.2 \mathrm{a}$ or $1.2 \mathrm{~b}$ become activated depending on the actual origin of Phobos. If

319 the moons are found to be of capture origin, then the formation and dynamical transport

320 of original bodies will be deciphered along with their cosmochemical characteristics

321 through sample analyses (1.2a). If they are found to be of giant impact origin, then the

322 timing and magnitude of the impact will be estimated along with the composition of the

323 impactor, which is indicative of its formation region, by sample analyses (1.2b). As for 
324 the Mars system evolution, MMX will reveal alteration processes of surface layers of

325 Phobos and Deimos (2.1), take new constraints on the Martian surface environment

326 evolution (2.2) and characterize the dynamical behavior of the Martian atmosphere that

327 relates to the atmospheric evolutionary processes (2.3). Processes such as possible dust

328 and/or gas torus formation along orbits and supply of Mars-derived rocky materials onto

329 Phobos may be unique for the Mars system.

$330 \quad$ (Table 3)

331

332 Considering that the MMX mission has been assembled from the primary objective of

333 exploring the Martian moons, the mission objectives are prioritized as shown by the

334 marks in Table 3. Determination of the origin of Phobos by spectroscopic observations

335 (MO1.1.1) and by sample analyses (MO1.1.2) has the highest priority as well as the

336 identification of weathering and evolutionary processes of Phobos surface (MO2.1.1).

337 Constraining Phobos' origin from its molecular release rate, mass distribution, and

338 surface density variation (MO1.1.3) is given high priority as well as revealing the

339 surface distribution of constituent materials in Deimos in comparison with those of 
340 Phobos (MO1.3.1). The second priority is also given for constraining the initial

341 conditions of celestial migration processes and evolution of the Martian surface from

342 predictions of the capture process (MO1.2a.1), and estimating the magnitude and timing

343 of the giant impact along with placing constraints for celestial migration and planetary

344 formation processes (MO1.2b.1). Objectives related to the observations of the Martian

345 atmosphere (MO2.2.2 and MO2.3.1) and the possible finding of particles ejected by

346 impact cratering on Mars since the formation of Phobos (MO2.2.1) are given third

347 priority.

349 Mission requirements

350

351 Corresponding to each mission objective, the mission requirements are deduced by

352 reflecting the current knowledge of the Martian moons and their parent planet (Table 3).

353 The detailed requirements concerning resolutions and accuracies for each type of

354 observation will be given by corresponding instrument papers. The general scientific

355 grounds for each mission requirement are described below. 
357 MR1.1.1 summarizes the requirements for remote sensing observations to constrain the

358 origin of Phobos. Previously obtained visible and near-infrared reflectance spectra of

359 Phobos are almost featureless with possible weak features indicative of olivine and

360 pyroxene (Gendrin et al. 2005) and phyllosilicates (Fraemann et al. 2014) but there

361 remain the limitation of $\mathrm{S} / \mathrm{N}$, spatial resolution, and/or coverage. If hydrated minerals

362 are proven to exist in fresh bedrock exposures, this provides strong evidence for the

363 capture origin of Phobos. Since the Phobos surface is possibly affected by space

364 weathering and late accumulated materials, it is valuable to determine reflectance

365 spectra for fresh materials exposed in and around young craters with diameters of about

$366100 \mathrm{~m}$ or larger. To achieve such observations, a spatial resolution of $20 \mathrm{~m}$ or better is

367 required for spectroscopic mapping of major areas on Phobos. High spatial resolution is

368 also valuable to determine the reflectance spectra of tens-m-size boulders which may

369 contain freshly exposed surfaces. Imaging with a spatial resolution of $1 \mathrm{~m}$ or better is

370 necessary for $\sim 50 \mathrm{~m}$ area including sampling site to characterize the geologic context of

371 samples. The mean $\mathrm{Si} / \mathrm{Fe}$ ratio on the hemisphere scale, which is observable by a 
372 gamma-ray spectrometer for instance, also provides an important clue to revealing

373 Phobos origin. If capture origin is the case, $\mathrm{Si} / \mathrm{Fe}$ ratio is expected to be within the range

374 of chondritic meteorites. If the giant impact origin is the case, $\mathrm{Si} / \mathrm{Fe}$ ratio may be $>20 \%$

375 larger than the chondritic range (1.1-2.0 Wasson and Kallemeyn 1988) due to the

376 contribution of Mars mantle materials.

377

378 MR1.1.2 describes the mission requirement for sampling and sample analyses to

379 determine Phobos' origin. To collect Phobos indigenous materials from the surface

380 possibly contaminated by exogenic materials, a large amount of granular sample is

381 necessary for statistical sorting by the isotopic composition. The average mixing ratio of

382 external materials is estimated to be several \% at most (Ramsley and Head 2013; Hyodo

383 et al. 2019). To distinct possible chemical diversity in indigenous materials and external

384 materials by statistical analyses, at least about one hundred particles with size applicable

385 for isotopic composition determination are necessary. The typical size of Phobos

386 regolith particles is estimated to range from $300 \mu \mathrm{m}$ to $2 \mathrm{~mm}$ (see section "Sampling

387 and Sample Analyses"). $300 \mu \mathrm{m}$ is enough for three oxygen isotope determination and 
$388 \sim 1 \mathrm{~mm}$ is enough for ${ }^{53} \mathrm{Cr} /{ }^{54} \mathrm{Cr}$ determination with sufficient accuracy (Usui et al. 2020).

389 Both isotope systems provide a key proxy to judge the type of the source body (Warren,

390 2011). Of course, complimentary application of textural, mineralogical, and chemical

391 analyses for grains is also useful to judge the origin of Phobos. This requires the sample

392 collection of $\geq 10 \mathrm{~g}$ samples for each sampling site. To obtain materials minimally

393 affected by space weathering caused by solar wind and meteoroid fluxes, samples

394 should include materials from a depth of $>2 \mathrm{~cm}$ that is deeper than the penetration

395 depth of solar wind (Nishiizumi et al. 2009). On the other hand, Phobos indigenous

396 materials may have compositional diversity as implied from the observed regional

397 difference in reflectance spectra represented by red unit and blue one (Rivkin et al.

398 2002; Fraeman et al. 2014). Here blue unit stands for areas having weaker reddening

399 trends, which may represent diversity in composition or weathering state on Phobos.

400 Therefore, samplings from at least two different sites are ideal. Also, to guarantee the

401 collected samples to be Phobos indigenous materials, material distribution on the entire

402 globe of Phobos as well as the geologic context of sampling sites should be constrained

403 by imaging and spectroscopic mapping. 
405 Mission requirements for the acquisition of data related to Phobos's internal structure

406 that independently provide constraints on Phobos origin are summarized in MR1.1.3.

407 The low density of Phobos compared to intact rocks possibly reflects the existence of

408 water ice in the deep interior or a significant porosity in this moon at all depths.

409 According to the thermal evolution model of Phobos (Fanale and Salvail 1990), the

410 emanation of water molecules may continue at present depending on the thermal and

411 molecular conductivities and assuming that Phobos was originally a primitive small

412 body bearing water ice. $10^{22}$ molecules/s is a possible emanation rate at present. The

413 emanation of $\mathrm{H}_{2} \mathrm{O}$ molecules, if detected, will strongly support the capture of an ice-

414 bearing small body for as Phobos' origin. The icy Phobos model also implies a

415 combination of a dense core made of a silicate-ice mixture and a porous silicate mantle

416 under the evaporative loss of ice from the surface. If mass concentration toward the

417 center is found from geodetic observation, it places important constraints on such a

418 model. 
421 MR1.2b mainly for sample analyses are given, respectively. If capture origin is the case

422 (MR1.2a), chemical, mineralogical and isotopic compositions are required to identify

423 the source region of the Phobos precursor. The application of chronological analyses in

424 combination with the geological survey is required for the estimation of time and

425 environment of the formation of the precursor body as well as for constraining the

426 impact history during the migration in the early Solar System. Volatile abundances in

427 Phobos indigenous materials are also necessary to evaluate how Phobos-like bodies

428 may contribute to built-up an ancient atmosphere-ocean-cryosphere system on proto-

429 Mars.

430

431 If giant impact origin is the case (MR1.2b), determination of textures, chemical,

432 mineralogical and isotopic compositions, and alteration ages of Phobos indigenous

433 materials are necessary for the estimation of the timing and magnitude of the impact

434 event, the chemical type of the impactor, as well as the mixing ratio of materials derived

435 from the impactor and proto-Martian mantle. To estimate the mixing ratio of the bulk 
moon fairly extending the sample constraints, measurement of elemental ratio such as

$437 \mathrm{Fe} / \mathrm{Si}$ is necessary for the Phobos surface material averaged over the hemisphere scale.

439 Mission requirements for constraining the origin of Deimos are described by MR1.3.1.

440 As for Deimos' observation, it is most important to confirm whether the genetic

441 commonality of Deimos and Phobos implied from the similar reflectance spectra at the

442 coarse spatial resolutions is the true character or surficial one. To achieve this, it is

443 necessary to obtain spectroscopic data constraining composition for major areas

444 containing widespread exposures of bedrocks of Deimos. A candidate area enriched in

445 such exposure is the Deimos's south pole basin, which is possibly the largest crater on

446 Deimos comparable to the Stickney crater of Phobos. However, the topography of this

447 basin has been poorly determined so far due to the lack of image at good illumination

448 conditions (Thomas 1993). A detailed study of the topography of this basin is necessary

449 also for the estimation of the Deimos's volume and thus density as well as for the

450 geological comparison of Deimos with Phobos. 
452 The mission requirements given by MR2.1 are specified for clarifying the surface

453 evolution and its processes of Phobos, paying attention to the difference in boundary

454 conditions from heliocentric small bodies. Since the Martian moons revolve around

455 Mars with relatively short periods, whenever an impact occurs, a considerable fraction

456 of ejecta particles that once escape the moons' gravity may re-impact onto the original

457 moon after revolutions around Mars. Such ejecta behavior may promote the thickening

458 of regolith layers of the moons (Ramsley and Head 2013) and cause the formation of

459 dust rings (or torus) along the satellite orbit through impact ejecta reproduction (Soter

460 1971). Since Phobos is positioned slightly outside the tidal break-up radius of Mars, it

461 may experience cratering events strongly influenced by the action of Martian tidal

462 forces and/or by ejecta from Martian craters (e.g. Basilevsky et al. 2014). The tidally

463 locked rotation may induce heterogeneous evolution of the surface layer between

464 leading and trailing hemispheres due to the different flux and approaching velocities of

465 impactors (Christou 2014). The molecular flux from the Martian atmosphere may affect

466 space weathering of the moons' surface along with the influx of solar wind and

467 meteoroids (e.g., Stofer 1971; Poppe and Curry 2014). To unravel those complex 
468 processes, it is necessary to conduct observations of dust and ion fluxes in circum-

469 Martian space, topography mapping with resolution enough for precise crater

470 chronology among major terrains and geomorphic features, and sample analyses

471 characterizing space weathering processes.

472

473 The mission requirements for the search for materials transferred from Mars after

474 Phobos formation in the returned sample are given by MR2.2.1. Based on the young

475 crater population on Mars and numerical simulations of impact processes, it is estimated

476 that Phobos regolith may contain on average at least $340 \mathrm{ppm}$ of ejecta materials from

477 Mars due to impacts during the last 500 Myr (Hyodo et al. 2019). The contaminated

478 ejecta materials likely consist not only of igneous rocks but also of sedimentary ones.

$47910 \mathrm{~g}$ of granular Phobos sample may contain several tens grains of such Martian ejecta

480 assuming that $300 \mu \mathrm{m}$ grains dominate the sample mass. Grain samples originated from

481 Martian terrains excavated by young impacts may allow material studies to characterize

482 the Martian surface environment at ages of terrain formation on Mars. To do so, it is

483 necessary to seek out impact ejecta materials from the returned samples and add 
484 adequate analyses to constrain ages and deposition environments on Mars.

485

486 The mission requirement given by MR2.2.2 is placed to conduct flux measurements of

487 major escaping ion species such as $\mathrm{O}^{+}$and $\mathrm{C}^{+}$with discriminating isotopes to improve

488 constraints on the isotopic fractionation in ion escape processes. Atmospheric escape is

489 one of the significant ongoing processes at Mars that have driven the evolution of the

490 Martian surface environment over a geologic time scale (e.g. Jakosky et al. 1994). The

491 isotopic fractionation of escaping species changes the residue volatile element isotope

492 values over geological time. Thus, the isotopic fractionation factor at escape is an

493 important parameter to interpret the isotopic difference among Martian volatile

494 reservoirs in terms of the time scale and magnitude of atmospheric loss. Past Martian

495 missions and ground-based observations have progressively revealed the isotopic

496 composition of volatile elements in the Martian atmosphere and sedimentary materials

497 along with analyses of Martian meteorites (e.g. Leshin et al. 2013; Mahaffy et al. 2013;

498 Villanueva et al. 2015, Jakosky et al. 2018). However, isotopic fractionation of escaping

499 elements, which probably depends on the level of solar activity, has been poorly 
501 2004).

503 The mission requirement given by MR2.3 is placed to realize monitoring atmospheric

504 flow and the transport of water and dust using the vantage of the equatorial orbit

505 moderately distant from Mars. The Martian atmosphere exhibits various meteorological

506 activities. Among them, atmospheric transport of water vapor and dust plays a

507 particularly important role in the evolution of the Martian climate system. The lateral

508 water transport is an elementary process for global-scale redistribution of water storage

509 of near-surface layer over the time scale of changes in obliquity and orbital parameters

510 such as eccentricity and the argument of periapsis caused by gravitational perturbations

511 by other planets (e.g. Mastard et al. 2001). Dust that distributes everywhere in the

512 Martian atmosphere with variable content significantly influences the radiative budget

513 of the thin atmosphere since dust is the primary absorber for visible and infrared

514 radiation (Pollack et al. 1979b). Therefore, dust strongly affects the atmospheric thermal

515 budget and dynamics, which in turn influence water vapor transport, including one 
516 across the vertical direction reaching the upper atmosphere where photolysis of water

517 molecules takes place (e.g. Heavens et al. 2018). However, it remains poorly

518 constrained how the dust transport is coupled with atmospheric dynamics due to the

519 paucity of continuous imaging observations that can capture developments of dust 520 events.

521

\section{Spacecraft and Science Instruments}

\section{Spacecraft}

524 Since the design of the MMX spacecraft architecture is reported in detail elsewhere

525 (Kawakatsu et al. 2019), here we briefly summarize its essence. The MMX spacecraft

526 will employ a chemical propulsion system with a high acceleration ability to confine the

527 roundtrip time to a reasonable length (Campagnola et al. 2018). To achieve a round trip

528 to the Martian sphere of gravity, the spacecraft is composed of separable modules:

529 propulsion module, exploration module, and return module (Figure 1). By releasing

530 used modules at appropriate epochs, the spacecraft mass will be reduced to allow orbital

531 controls in the Martian system and return cruise to the Earth reducing the required 
532 overall propellant mass. To carry samples with a volume much larger than that of

533 Hayabusa2 which aims to take $100 \mathrm{mg}$ of samples (Tsuda et al. 2013), an enlarged

534 return capsule will be used for MMX. The total spacecraft wet mass at launch is about

$5354,000 \mathrm{~kg}$. The spacecraft will be launched by an H-III rocket with launch capabilities

536 larger than H-II rockets. The H-III rocket system is under development as the new

537 Japanese flagship launch vehicle with the planned first launch in 2021.

$538 \quad$ (Figure 1)

539

\section{Science instruments}

541 Table 4 lists the science instruments aboard MMX. These are selected to satisfy the

542 mission requirements complimentary to the sample analyses. As the detailed description

543 of each instrument will be given elsewhere, here we summarize their complementary

544 roles.

545 (Table 4)

546

547 One of the most important roles of proximity observations is to obtain constraints for 
548 the origin of the Martian moons independently of sample analyses and also to document

549 the sampling site to inform the geological context. Measurements for the determination

550 of bulk Phobos composition is the most direct approach for this purpose. OROCHI

551 (Kameda et al. this issue), the wide-angle visible multiband camera, and MIRS (Barucci

552 et al. this issue.), the infrared spectrometer, will conduct near-global mapping of

553 reflectance spectra of Phobos with spatial resolutions of the order of 10 meters.

554 Combining with the specification of fresh surface exposures by TENGOO (Kameda et

555 al. this issue), a panchromatic telescopic camera with much higher spatial resolutions,

556 the spectroscopic mapping over wavelength from visible to infrared will constrain the

557 mineral composition of Phobos bedrock. Hydrous minerals are a very important proxy

558 indicative of the captured origin of the moon, thus OROCHI and MIRS are designed to

559 detect absorption bands at $\sim 0.7 \mu \mathrm{m}$ and $\sim 2.7 \mu \mathrm{m}$, respectively, caused by hydroxyl

560 group in mineral structures with sufficient signal to noise ratio.

561

562 MEGANE (Lawrence et al. 2019), the gamma-ray and neutron spectrometer will

563 determine the concentration of elements such as $\mathrm{Si}, \mathrm{Fe}, \mathrm{H}$, and $\mathrm{K}$ in the surface layer of 
several tens $\mathrm{cm}$ thickness, where cosmic-ray induced and spontaneous atomic nucleus reactions take place associated with emissions of neutrons and high energy photons to space, over the hemisphere scale. MSA (Yokota et al. this issue.), the ion mass spectrum

567 analyzer, will attempt to detect the ion torus derived from $\mathrm{H}_{2} \mathrm{O}$ possibly released from

568 Phobos which may store icy materials inside. Metallic ions spattered out from the

569 surface of Phobos by solar wind are also the target of the MSA measurements, which

570 may constrain the elemental composition of Phobos surface averaged over the

571 hemisphere scale as is the case for the Earth`s moon (Yokota et al. 2009).

573 LIDAR (Senshu et al. this issue), the laser altimeter, will be used for the determination

574 of the shape, rotation, and gravity field of Phobos in combination with the topographic

575 imaging by TENGOO and Earth-based spacecraft tracking data. Combining the

576 rotational quantity with the gravity field determination based on precise analyses of

577 inertial spacecraft motion around Phobos, one can deduce constraints on Phobos's

578 internal density distribution such as the moment of inertia factor. A shape model

579 provides the position of the center of mass relative to the geometrical center. These may 
reflect possible compositional heterogeneity of this moon, for instance, the presence of

581 interstitial ice in the deep interior, the presence of porosity, or the coalescence of

582 planetesimals with different compositions. LIDAR will also provide surface roughness

583 information within each laser footprint from analysis of the reflectance as a function of

584 observation angle at the laser wavelength of $1064 \mathrm{~nm}$.

585

586 Those remote sensing data will be also used for the selection of sampling sites that are

587 required to be sufficiently flat for safe landing and accessibility to Phobos indigenous

588 materials. The global surface composition of Phobos constrained by proximity

589 observations will be compared with the compositions of the returned sample to examine

590 how returned materials are representative of Phobos indigenous materials. Close-up

591 spectroscopic and panchromatic imaging by OROCHI and TENGOO with spatial

592 resolutions in the order of mm during landing operations will be used to describe the

593 geologic context of the sampling site, which is necessary to interpret the possible

594 variation of composition and grain size, texture, and weathering state of returned samples. 
597 The topographic and compositional mapping data will be also used to elucidate the

598 geologic processes that have evolved the surface of Phobos in the areocentric orbit.

599 Crater counting with higher spatial resolution provides a basis for its resurfacing

600 history. CMDM (Kobayashi et al. 2018) is a dust detector monitoring impact signals on

601 its sensor of an exposed thin film at the top layer of multi-layer insulation of the

602 spacecraft. It will measure the impact momentum of dust particles colliding with the

603 sensor surface in orbit around Mars. When there is a dust collision on the sensor, it is

604 classified as interplanetary dust, interstellar dust, or Mars orbiting dust based on the

605 information on the orbital position and attitude of the spacecraft at that time. As a result,

606 the flux of those dust species is obtained. Confirmation of the presence or absence of a

607 hypothesized dust ring or torus along the moons' orbits is one of the main purposes of

608 this measurement. Furthermore, the measured fluxes of dust particles including

609 meteoroids provide basic data for understanding the weathering and impact gardening

610 processes of both moons. By combining the analyses of samples concerning impact

611 alteration states and ages, mixing of exotic materials, and possible material exchange 
612 among bodies in the Martian system, an integrated view for the surface evolution of the

613 Martian moons would be available.

614

615 OROCHI and MIRS will also conduct a series of spectroscopic imaging/mapping of the

616 atmosphere of Mars. From positions near Phobos, both instruments are capable of

617 imaging a wide area from mid to low latitudes of Mars. At positions more distant from

618 Mars, imaging of the global hemisphere becomes easier. Color imaging by OROCHI

619 can identify ice clouds and atmospheric dust, whereas infrared spectroscopic imaging

620 by MIRS can assess the distribution of the column $\mathrm{H}_{2} \mathrm{O}$ vapor content using the

621 reflectance contrast between the wavelengths in and outside the $\mathrm{H}_{2} \mathrm{O}$ absorption band.

622 By tracing the motion of clouds, atmospheric dust swarms, and $\mathrm{H}_{2} \mathrm{O}$ vapor using the

623 time series of imaging/spectroscopic data, the atmospheric circulation can be

624 understood and the exchange of $\mathrm{H}_{2} \mathrm{O}$ among the surface reservoirs can be constrained.

625 Additionally, zoom-up observations by TENGOO would capture the upwelling of dusty

626 warm air into the upper atmosphere, which may be a key process promoting $\mathrm{H}_{2} \mathrm{O}$

627 photolysis and $\mathrm{H}$ escape to space (Heavens et al. 2018). Ions escaping from the Martian 
upper atmosphere are detectable by MSA with distinguishing isotopic mass of elements

629 including $\mathrm{O}$ and $\mathrm{C}$. This would provide us better constraints on isotopic fractionation in

630 the atmospheric escape processes. From those observations of the Mars atmosphere, one

631 can reveal atmospheric processes that have driven the evolution of the Martian surface

632 environment.

633

634 MMX will carry a rover provided by CNES and DLR for scientific and engineering

635 purposes including being a scout for the lander (Michel et al. this issue). The landing

636 system and landing operation of the mothership have been studied to absorb the

637 uncertainties in the mechanical properties of Phobos surface regolith such as the surface

638 layer strength. On the other hand, it is beneficial to confirm in advance whether the

639 actual regolith properties are within or outside the expected range. The rover will be

640 released before the landing operation and conduct an in-situ examination of the Phobos

641 surface. The rover powered by its solar generator will be equipped with navigation

642 cameras, wheels for travel with torque sensors, wheel cameras, imaging the interaction

643 of the wheels with the regolith, a thermal radiation monitor (MiniRad), accelerometers 
644 characterizing impact and bouncing, and a laser Raman microscope, RAX (Cho et al.

645 this issue). These instruments will examine mechanical, dynamical, and thermal

646 properties and mineralogy of Phobos surface regolith. The obtained data are also useful

647 as ground truth for interpreting the remote sensing data taken from orbit.

648

649 The sampling system, one of the most important mission instruments of MMX, will

650 conduct sampling from Phobos surface regolith and transfer of samples into a sample

651 container inside the sample return capsule (Kawakatsu et al. 2019). After touch down,

652 sampling will be conducted by using a joint arm manipulator equipped with a coring

653 mechanism at its tip. The MMX mission is planned to make landings at two different

654 sites. At each landing site, a sampling point will be first determined within the area

655 accessible by manipulation based on a precise raw surface image transmitted to the

656 ground station on Earth. An installed corer with an inner diameter of $2 \mathrm{~cm}$ will penetrate

657 to a sufficient depth at the designated point and then the inner sample holder will be

658 transferred to the container space in the return capsule. OROCHI will conduct

659 spectroscopic imaging of the sampling point and its surroundings with a spatial 
660 resolution of the order of $\mathrm{mm}$ to specify their spectroscopic property and geologic

661 context which are important to assess how the acquired samples are representative of

662 Phobos indigenous materials.

663

664 A pneumatic sampler, another type of sampler provided by NASA applying the same

665 working principle as used during the OSIRIS-Rex mission (Bierhaus et al. 2018),

666 installed at a landing pad will also attempt sampling of regolith at shallow depth

667 (Kawakatsu et al. 2019). Samples taken from different depths are useful to understand

668 the possible layering of surface regolith caused by space weathering, the influx of exotic

669 materials, and gardening by meteoroid impacts (Usui et al. 2020). We call this sampler

670 P-sampler whereas the primary coring sampler is called C-sampler. The sample holder

671 of the P-sampler will be transported to the container space in the return capsule by the

672 manipulator.

673

674 Mission Profile

675 Overall 
676 The planned mission profile is shown in Figure 2. The MMX spacecraft is planned to be

677 launched in September 2024 and reach the Martian system after the cruise slightly less

678 than a year. The spacecraft will stay in the Martian system for nearly 3 years and

679 conduct close-up observations of both moons, delivery of the rover, and sampling from

680 Phobos. The spacecraft will depart the Martian system in August 2028 and return the

681 capsule containing collected samples to the Earth.

682 (Figure 2)

683

684 After the insertion to a circum-Martian orbit, the spacecraft motion will be tuned to

685 approximate the Phobos motion. By taking small differences in orbital eccentricity from

686 Phobos, the spacecraft will eventually circulate Phobos at small distances. In the frame

687 fixed to Phobos, the spacecraft trajectory will form an ellipse, or quasi-satellite-orbit

688 (QSO), with the center at the Phobos mass center (Figure 3). The semimajor axis of

689 QSO, which is perpendicular to the Mars direction, is about twice of the minor axis.

690 Decreasing in separation from Phobos, QSO gradually approaches to circle, or the

691 semimajor axis/minor axis ratio decreases, due to the greater contribution of Phobos 
692 gravity. MMX will utilize several QSO patterns with high (semimajor axis $200 \mathrm{~km}$,

693 QSO-H), medium (100 km, QSO-M), and low (50 km and below, QSO-L series)

694 altitudes depending on the observation phase. MMX will also introduce the so-called

695 3D-QSO with inclination to Phobos, which allows observation of high latitude regions

696 of this moon (Figure 3b). From QSOs, MMX will carry out observations of Phobos

697 including those for landing site selection, circum-Martian space, and the Martian

698 atmosphere.

699 (Figure 3)

700

701 During the period in proximity to Phobos, the spacecraft will land on two different sites

702 of Phobos. Since Phobos's surface gravity is two orders of magnitude larger than those

703 of Itokawa and Ryugu, the descent and landing sequence of MMX spacecraft is

704 different from the prior missions Hayabusa and Hayabusa2 which used a so-called

705 touch and go approach for sampling operations. For example, a low-velocity descent

706 from a very high altitude cannot be adopted considering fuel consumption to keep low

707 velocity against gravity. Instead, from low QSO orbit, a ballistic descent will be applied 
708 to reach $\sim 2 \mathrm{~km}$ above a landing site before starting the subsequent final vertical descent.

709 A rehearsal operation before landing will take close-up images of the area including

710 landing site candidates to confirm the safety and the availability of samples that are

711 representative of bedrock materials. To avoid previously unrecognized obstacles such as

712 large boulders in the area of the selected site, an autonomous hazard detection and

713 avoidance technology will also be set up to realize a safe landing. The landing gear is

714 installed with a mechanism absorbing the spacecraft momentum relative to the surface

715 to prevent the spacecraft from bouncing at touch down.

716

717 After the completion of proximity observations of Phobos and sampling operations, the

718 spacecraft will expand its orbit to perform multiple flybys of Deimos. During this

719 period, close-up observations of Deimos will be conducted together with those of the

720 surrounding space and the Martian atmosphere. Then, the spacecraft will depart from

721 the Martian system.

722

723 Operations during the stay around the Martian moons 
725 In the nominal operation plan, solar conjunctions will occur in January 2026 and March

7262028 during the stay around Mars. Science operations may be inhibited for $\sim 1.5$ months

727 around these conjunction timings. Furthermore, Mars will arrive at equinoxes in late

728 November 2025 and this will happen subsequently after every about half of the Martian

729 year. During about three months with each conjunction timing in the middle, low QSOs

730 around Phobos will experience eclipses with long duration by Mars and Phobos

731 repeatedly. This is caused by the near-equatorial orbit of Phobos around Mars and

732 relatively small differences in QSO periods from the length of the day of Phobos. For

733 maintaining the health of electric devices, long stops of power generation should be

734 avoided. During those periods around equinoxes, therefore, QSO operation is restricted

735 to large ecliptic orbit with minor and major radii no smaller than $50 \mathrm{~km}$ and $100 \mathrm{~km}$

736 around Phobos to reduce the duration of every eclipse.

737

738 The current plan for observational operation is shown in detail elsewhere (Nakamura et

739 al. this issue), here we briefly overview it below. Considering the constraints shown 
740 above, the period of $\sim 3$ years stay in the sphere of Martian gravity is divided into five

741 phases as shown in Figure 4. Period 1 starts from MOI (Mars Orbit Insertion). After

742 MOI, the spacecraft will be transferred to QSO around Phobos after the deployment of

743 the propulsion module. During this transfer, the spacecraft will have a chance to

744 approach Deimos at outer orbit, which would be used as an opportunity for the first

745 Deimos imaging observation from small distances. After insertion to QSO, observations

746 of Phobos from QSO with larger orbital radii (QSO-H) will be conducted for the near-

747 global shape model generation and spectral mapping of Phobos with a typical spatial

748 resolution of several meters for telescopic monochrome imaging and several 100 meters

749 for spectroscopic imaging by OROCHI and MIRS. At this time, the LIDAR footprint

750 will be several $10 \mathrm{~m}$ from QSO-H.

751

752 During phase 2 that starts after the first solar conjunction, close-up observations from

753 QSOs with minor and major radii as small as $30 \mathrm{~km}$ and $50 \mathrm{~km}$ or even below will be

754 combined to conduct higher resolution imaging and spectral mapping up to typical

755 spatial resolution $\sim 40 \mathrm{~cm}$ and $\sim 20 \mathrm{~m}$, respectively. The LIDAR footprint will become as 
small as several $\mathrm{m}$. Observations from QSO with low radii are mandatory for the

757 gamma-ray and neutron spectrometry by MEGANE which needs a large solid angle of

758 the targeting body in the field of view to reduce sufficiently the effect of background 759 noise.

760

761 The descent and landing operations will be performed during phase 3 using the

762 advantage in solar illumination conditions and short Earth-Mars distance. The release of

763 the rover and the descent and ascent operation, which is a part of the landing rehearsal,

764 will be done before the first actual landing but their best timings are under study

765 including the last part of phase 2. Observations with progressive improvement of spatial

766 resolution during phases 1 and 2 will be used for the landing site selection to guarantee

767 the safety for landing and the accessibility to indigenous materials. MMX plans to

768 perform sampling from multiple landing sites because Phobos is known to have a

769 composite surface made of units with colors distinguished by slopes of reflectance

770 spectra as mentioned above. 
772 A landing operation that includes touch-down, sampling, and departure will be

773 completed during a single daytime of Phobos lasting 3 hours 50 minutes. The stay time

774 on the surface is planned to be 2.5 hours with about a 1-hour margin in the entire

775 daytime. The time duration allocated for the sampling operation is 1.5 hours, which

776 includes close-up imaging of the landing area to determine the sampling point by the C-

777 sampler and the telemetries of image data and command with the control station on the

778 Earth. During the first landing, the P-sampler system whose nozzle is equipped on a

779 landing pad will be used.

780

\section{Sample Sciences}

782

783 Due to the current lack of direct measurement, the grain size distribution of Phobos

784 regolith remains uncertain. According to the interpretation of Phobos thermal inertia

785 from the modeling of thermal conduction through granular layers, the typical grain size

786 in Phobos regolith is estimated to be $\sim 1 \mathrm{~mm}$ (Gundlach and Blum 2013). An expanded

787 method incorporating the physical relationships between porosity and grain size under 
788 the action of intra-grain cohesion forces and Phobos's gravity suggests the typical size

789 of $\sim 2 \mathrm{~mm}$ (Kiuchi and Nakamura 2014). Among ejecta released from the Phobos

790 surface by impacts, grains smaller than $\sim 300 \mu \mathrm{m}$ would be preferentially lost to the

791 parent planet or outer space due to the action of solar radiation pressure (Ramsley and

792 Head 2013) while large grains may return onto the surface of Phobos after areocentric

793 motion. For those reasons, the regolith grains are likely to have sizes mainly between

$794 \sim 300 \mu \mathrm{m}$ and $\sim 2 \mathrm{~mm}$. MMX will collect granular samples of more than $10 \mathrm{~g}$ from each

795 landing site, and is expected to obtain grains in numbers of the order of thousands or

796 more. The acquisition of a large number of regolith grains will enable us to apply

797 statistical analyses to deduce an estimate for the bulk composition of Phobos even when

798 grains exhibit a wide variation in their composition.

799

800 Analyses of returned samples will include those on grain size, shape, texture,

801 mineralogy, chemistry, isotopic composition, microscopic weathering state, chronology,

802 and so on. These measured properties will provide a set of ground truth data for the

803 global compositional map acquired by MMX remote sensing data. Sample analyses will 
804 then provide us the best clue to judge the origin of Phobos as well as the processes of

805 Phobos formation in the context of material transport in the early Solar System and

806 those of Phobos surface evolution. Not only the records of shock alteration but also

807 possible extraction of exotic materials from samples are expected to provide us unique

808 information on the evolution of the Martian system including the Martian surface

809 environment.

810

811 If the majority of sampled Phobos indigenous materials are like unequilibrated

812 chondritic materials, this provides strong evidence for the capture origin of Phobos. In

813 this case, the sample is expected to be abundant in grains composed of unequilibrated

814 minerals enriched in volatile elements. Comparison of mineralogical, compositional,

815 and isotopic evidence with primitive meteorites will tell us the genetic relationships of

816 Phobos precursor and parent bodies of primitive meteorite groups in the early Solar

817 System. Using the corresponding relationships among the spectroscopic taxonomy of

818 asteroids and meteorite groups (DeMeo and Carry 2014), the source region of the

819 Phobos precursor may be estimated. Reflectance spectra of returned samples that 
contain less altered materials may also provide better constraints on the source asteroid

821 type of the Phobos precursor. Adding to the precise impact crater statistics that will be

822 revealed by MMX close-up observations, the distribution of shock alteration ages of

823 grains will provide constraints on the major impact history of Phobos including the

824 stage of its precursor body with heliocentric orbit. With help of models for the

825 dynamical evolution of small bodies in the early Solar System, the mechanisms and

826 history of transport of $\mathrm{H}_{2} \mathrm{O}$-bearing bodies from the early outer Solar System to

827 terrestrial planets will be elucidated. Furthermore, processes that formed $\mathrm{H}_{2} \mathrm{O}$-bearing

828 bodies in the early outer Solar System may be clarified from the analyses of minerals

829 produced by aqueous alteration.

830

831 If the moon is formed by a giant impact, Phobos's indigenous materials likely have

832 textures and compositions reflecting high-temperature melting and partial vaporization

833 induced by the giant impact event. Such an event could occur with an impact velocity

834 not much larger than the escape velocity of Mars, and materials ejected into areocentric

835 orbits may inefficiently be mixed in grain-scale due to rapid solidification by efficient 
836 radiative cooling (Hyodo et al. 2017). In this case, Phobos indigenous materials may

837 show isotopic and chemical compositions distributed on a mixing line connecting the

838 compositions of the proto Martian mantle and the impactor. Since the isotopic

839 compositions of elements such as oxygen are already known for Mars from the analyses

840 of Martian meteorites, the direction of the end of the mixing line points to the impactor

841 isotopic composition from which the source region of impactor may be estimated.

842 Materials that fall on isotopic end members could also tell us the chemical composition

843 of the proto Martian mantle and the impactor. The range of compositional dispersion

844 varies with the degree of material mixing, which reflects the magnitude of the moon-

845 forming impact. Crystallization ages of sample materials would constrain the timing of

846 giant impact which should be compared with the cratering record, especially of large

847 impact basins on Mars. If the impactor is found to be a volatile-rich body from the

848 sample isotopic signatures, its contribution to the development of the Martian

849 atmosphere and hydrosphere can be estimated. This should improve our understanding

850 of how Mars acquired atmosphere and water, for instance, regarding the significance or

851 insignificance of late accreting bodies. On the other hand, the composition of the proto- 
852 Martian mantle estimated from sample analyses would provide us valuable information

853 on the chemical state of the early Martian mantle at the timing of the moon-forming

854 impact.

855

856 Phobos surface materials have likely been influenced by fluxes of external materials:

857 heliocentric small bodies with various sizes, impact ejecta from Mars and Deimos, and

858 ions originating from the solar wind and Martian atmosphere. Self-reproductive

859 areocentric dust particles may also continuously hit the Phobos surface. Analyses of

860 sample grains focusing on alteration by these processes would reveal how Phobos

861 surface, probably poor in endogenic activities that affect material properties, has

862 evolved in the circum-Martian environment. For example, a histogram of shock

863 alteration ages of sample grains, likely measurable by Ar-Ar dating, may provide

864 information on the history of high-speed, large-scale impacts onto Phobos along the

865 lines of similar studies for the Earth's Moon (e.g. Fernandes et al. 2013).

866 Concentrations of implanted solar wind ion in weathered samples, as well as cosmic-ray

867 exposure age, may be used to estimate the resurfacing time scale of Phobos (e.g. Nagao 
et al. 2011 for Itokawa). The concentration of exotic materials would include

869 information on the ejecta transport from Mars.

870

871 If fragments of younger impact ejecta from Mars are identified in the sample grains,

872 they would provide information on the state of the surface environment on Mars at the

873 timing of bedrock formation at impact site(s) (Usui et al. 2020). Their mineral

874 assemblages, isotopic compositions, and magnetization are potential measurable proxies

875 for the past surface environment of Mars. To make them even more valuable, the age

876 determination of corresponding sample grains is quite important. Our capability to do

877 this depends on grain size and mineral composition. If such measurements are

878 successful for ejecta grains of various ages, it would significantly improve our

879 understandings of the evolution of Mars.

880

881 Concluding Remarks

882

883 Through the exploration of the Marian moons, the MMX mission extends the 
experience in Japanese sample return exploration for the small bodies and will investigate key processes for the formation and evolution of habitable planets having

886 atmosphere and water. Close-up observations of both moons and detailed analyses of

887 samples returned from Phobos will characterize the properties of their constituent

888 materials in great detail. The mission will determine the origin of the moons which is

889 currently under debate, with models of the capture of primordial, carbonaceous

890 asteroids and the accumulation of circum-Martian debris ejected by a huge impact on

891 early Mars both being seriously considered. Also, processes for the acquisition of water,

892 organics, and volatiles from the outer Solar System by early terrestrial planets will be

893 elucidated. If the giant impact origin is the case, the differentiation state of a proto-

894 planet mantle just before the occurrence of the moon forming impact will also be a

895 study target.

896

897 Sample analyses will also extract records on the long-term evolutionary processes such

898 as continuous impactor flux and irradiation of solar wind, cosmic rays, and circum-

899 Martian plasma. For this issue, it is beneficial to conduct multidisciplinary studies with 
900 remote and in-situ data including the crater population and surface reflectance of the

901 moons and the dust and plasma environment in circum-Martian space. Concurrent

902 evolutionary processes such as atmospheric circulation and escape and the possible

903 formation of dust ring and gas torus along the moons' orbits will also be study targets.

904 Ejecta materials derived from young Martian craters, if found in the returned samples,

905 will provide unique information on the Martian surface environment at the time of

906 impact and/or bedrock formation before impact.

907

908 The status of the MMX mission is now in phase B as a project of JAXA. Targeting the

909 launch in 2024, the design of spacecraft systems and instruments as well as studies of

910 spacecraft orbits, operations, and data processing including basic experiments are

911 extensively ongoing.

912 


\section{Declarations}

\section{Availability of data and materials}

915 Not applicable.

\section{Competing interests}

917 The authors declare that they have no competing interests.

\section{$918 \quad$ Funding}

919 Through the Phase-A and Phase-B activities of MMX, our work was supported by

920 ISAS/JAXA, NASA, CNES, DLR, and ESA. Parts of studies are also supported by

921 Grants-in-Aid from the Japan Society for the Promotion of Science to KK, HG, and TU

922 (18K03719, 17H06457, and 17H06459).

\section{Authors' contributions}

924 Overall conceptualization: KK, YK, MF, TU, and KW. Science backgrounds: GH, TI,

925 KM, HM, TM, TN, KO, NT, and SW. Origin of Phobos and Deimos: TN and HG.

926 Surface science and geology: HM and KW. Mars science: TI, NT, and HN. Geodesy:

927 KM and HS. Spacecraft and instrument system: YK, HO, KO, and MO. LIDAR: HS

928 (CIT) and KM. TENGOO/OROCHI: SK and MO. MIRS:MAB, HN, and TN, 
930 and SU. Sampler: HS (jaxa) and TU. Scientific operation and orbital design: TN, HS

931 (CIT), and HI. Sample sciences: TU, ST, and SR. All authors discussed the study and

932 commented on the manuscript.

933

\section{Acknowledgements}

935 We thank the MMX study team, the MMX project team, the sub-science teams (Origin

936 of Phobos and Deimos, Early Solar System Evolution, Surface Science and Geology,

937 Mars Science, and Geodesy), the instrument teams (CMDM, C-Sampler, LIDAR,

938 MIRS, MEGANE, MSA, ROVER, and TENGOO-OROCHI), and the working teams

939 (Data Processing, Landing Operation, Landing Site Selection, Mission Operation,

940 Rover Operation, and Sample Analysis) for insightful discussions for this project. 


\section{References}

943 Barruci MA, Reess JM, Bernardi P, Doressoundiram A; Fornaiser S; Du ML, Iwata T,

944 Nakagawa H, Nakamura T, André Y, Aoki S, Arai T, Baldit E, Beck P, Buey JT,

945 Canalias E, Castelnau M, Charnoz S, Chaussidon M, Chapron F, Ciarletti V, Delbo M,

946 Dubois B, Gauffre S, Gautier T, Genda H, Hassen-Khodja, R; Hervet G, Hyodo R,

947 Imbert C, Imamura T, Jorda L, Kameda S, Kouach D, Kouyama T, Kuroda T,

948 Kurokawa H, Lapaw L, Lasue J, Deit LL, Ledot A, Leyrat C, Ruyet BL, Matsuoka M,

949 Merlin F, Miyamoto H, Moynier F, Tuong NN, Ogohara K, Osawa T, Parisot J, Pistre

950 L, Quertier B, Raymond S, Rocard F, Sakanoi T., Sato TM, Sawyer E, Tache F,

951 Trémolières S, Tsuchiya F, Vernazza P, Zeganadin D, MIRS an Imaging spectrometer

952 for the MMX mission, This issue.

953 Basilevsky AT, Lorenz CA, Shingareva TV, Head JW, Ramsley KR, Zubarev AE

954 (2014) The surface geology and geomorphology of Phobos. Planetary and Space

955 Science, 102, 95-118. doi:10.1016/j.pss.2014.04.013

956 Bibring JP, Langevin Y, Mustard JF, Poulet F, Arvidson R, Gendrin A, Gondet B,

957 Mangold N, Pinet P, Forget F, Berthe M, Bibring JP, Gendrin A, Gomez C, Gondet B, 
958 Jouglet D, Poulet F, Soufflot A, Vincendon M, Combes, M, Drossart P, Encrenaz T,

959 Fouchet T, Merchiorri R, Belluci G, Altieri F, Formisano V, Capaccioni F, Cerroni P,

960 Coradini A, Fonti S, Korablev O, Kottsov V, Ignatiev N, Moroz V, Titov D, Zasova L,

961 Loiseau D, Mangold N, Pinet P, Doute S, Schmitt B, Sotin C, Hauber E, Hoffmann H,

962 Jaumann R, Keller U, Arvidson R, Mustard JF, Duxbury T, Forget F, Neukum G (2006)

963 Global mineralogical and aqueous Mars history derived from OMEGA/Mars Express

964 data. Science, 312, 400-404. doi:10.1126/science.1122659

965 Burns JA (1978) The dynamical evolution and origin of the Martian moons. Vistas in

966 Astronomy, 22, 193-210. doi:10.1016/0083-6656(78)90015-6

967 Bierhaus EB, Clark BC, Harris JW, Payne KS, Dubisher RD, Wurts DW, Hund RA,

968 Kuhns RM, Linn TM, Wood JL, May AJ, Dworkin JP, Beshore E, Lauretta DS,

969 OSIRIS-REx Team (2018) The OSIRIS-REx spacecraft and the touch-and-go sample

970 acquisition mechanism (TAGSAM). Space Science Reviews, 214, 107.

971 doi:10.1007/s11214-018-0521-6.

972 Campagnola S, Yam CH, Tsuda Y, Ogawa N, Kawakatsu Y (2018) Mission analysis for

973 the Martian Moons Explorer (MMX) mission. Acta Astronautica, 146, 409-417. 
975 Canup R, Salmon J (2018) Origin of Phobos and Deimos by the impact of a Vesta-to-

976 Ceres sized body with Mars. Science advances, 4, eaar6887.

977 doi:10.1126/sciadv.aar6887

978 Chassefière E, Leblanc F (2004) Mars atmospheric escape and evolution; interaction

979 with the solar wind. Planetary and Space Science, 52, 1039-1058.

980 doi:10.1016/j.pss.2004.07.002

981 Carr MH (1996) Water on Mars. New York: Oxford University Press.

982 Cho Y, Böttger U, Rull, F, Belenguer T, Börner A, Buder M, Bunduki Y, Dietz E,

983 Hagelschuer T, Hübers H-W, Kameda S, Kopp E, Lieder M, Lopez G, Moral Inza A,

984 Paproth C, Perez Canora C, Pertenais M, Peter G, Prieto Ballesteros O, Rockstein S,

985 Rodd-Routley S, Rodriguez Perez P, Ryan C, Santamaria P, Säuberlich T, Schrandt F,

986 Schröder S, Stangarone C, Ulamec S, Usui T, Weber I, Westerdorff K, Kuramoto K, In-

987 situ science on Phobos with the Raman spectrometer for MMX (RAX) onboard the

988 MMX rover. This issue

989 Christou AA, Oberst J, Lupovka V, Dmitriev V, Gritsevich M (2014) The meteoroid 
990 environment and impacts on Phobos. Planetary and Space Science, 102, 164-170.

991 doi:10.1016/j.pss.2013.07.012

992 Dauphas N, Pourmand A (2011) Hf-W-Th evidence for rapid growth of Mars and its 993 status as a planetary embryo. Nature, 473, 489-492. doi:10.1038/nature10077

994 Debaille V, Brandon AD, Yin QZ, Jacobsen B (2007) Coupled ${ }^{142} \mathrm{Nd}--^{143} \mathrm{Nd}$ evidence 995 for a protracted magma ocean in Mars. Nature, 450, 525-528. doi:10.1038/nature06317 996 DeMeo FE, Carry B (2014) Solar System evolution from compositional mapping of the 997 asteroid belt. Nature, 505, 629-634. doi:10.1038/nature12908

998 Duxbury TC, Zakharov AV, Hoffmann H, Guinness EA (2014) Spacecraft exploration 999 of Phobos and Deimos. Planetary and Space Science, 102, 9-17.

1000 doi:10.1016/j.pss.2013.12.008

1001 Fanale FP, Salvail, JR (1990) Evolution of the water regime of Phobos. Icarus, 88, 380-

1002 395. do:10.1016/0019-1035(90)90089-R

1003 Fernandes VA, Fritz J, Weiss BP, Garrick-Bethell, I, Shuster DL (2013) The

1004 bombardment history of the Moon as recorded by ${ }^{40} \mathrm{Ar}-{ }^{39} \mathrm{Ar}$ chronology. Meteoritics

1005 and Planetary Science, 48, 241-269. doi:10.1111/maps.12054 
1006 Fraeman AA, Murchie SL, Arvidson RE, Clark RN, Morris RV, Rivkin AS, Vilas F

1007 (2014) Spectral absorptions on Phobos and Deimos in the visible/near infrared

1008 wavelengths and their compositional constraints. Icarus, 229, 196-205.

1009 doi:10.1016/j.icarus.2013.11.021

1010 Fujiya W, Sugiura N, Hotta H, Ichimura K, Sano Y (2012) Evidence for the late

1011 formation of hydrous asteroids from young meteoritic carbonates. Nature

1012 communications, 3, 627. doi:10.1038/ncomms1635

1013 Fujiwara A, Kawaguchi J, Yeomans DK, Abe M, Mukai T, Okada T, Saito J, Yano H,

1014 Yoshikawa M, Scheeres DJ, Barnouin-Jha O, Cheng AF, Demura H, Gaskell RW,

1015 Hirata N, Ikeda H, Kominato T, Miyamoto H, Nakamura AM, Nakamura R, Sasaki S,

1016 Uesugi K (2006) The rubble-pile asteroid Itokawa as observed by Hayabusa. Science,

1017 312, 1330-1334. DOI: 10.1126/science.1125841.

1018 Genda H (2016) Origin of Earth's oceans: An assessment of the total amount, history

1019 and supply of water. Geochemical Journal, 50, 27-42. doi:10.2343/geochemj.2.0398

1020 Gendrin A, Langevin Y, Erard S (2005) ISM observation of Phobos reinvestigated:

1021 Identification of a mixture of olivine and low-calcium pyroxene. Journal of Geophysical 
1023 Greeley R (1987) Release of juvenile water on Mars: Estimated amounts and timing

1024 associated with volcanism. Science, 236, 1653-1654.

1025 doi:10.1126/science.236.4809.1653

1026 Grotzinger JP, Sumner DY, Kah LC, Stack K, Gupta S, Edgar L, Rubin D, Lewis K,

1027 Schieber J, Mangold N, Milliken R, Conrad PG, DesMarais D, Farmer J, Siebach K,

1028 Calef F, Hurowitz J, McLennan SM, Ming D, Vaniman D, Crisp J, Vasavada A, Edgett

1029 KS, Malin M, Blake D, Gellert R, Mahaffy P, Wiens RC, Maurice S, Grant JA, Wilson

1030 S, Anderson RC, Beegle L, Arvidson R, Hallet B, Sletten RS, Rice M, Bell, J, Griffes J,

1031 Ehlmann B, Anderson RB, Bristow, TF, Dietrich WE, Dromart G, Eigenbrode J,

1032 Fraeman A, Hardgrove C, Herkenhoff K, Jandura L, Kocurek G, Lee S, Leshin LA,

1033 Leveille R, Limonadi D, Maki J, McCloskey S, Meyer M, Minitti M, Newsom H,

1034 Oehler D, Okon A, Palucis M, Parker T, Rowland S, Schmidt M, Squyres S, Steele A,

1035 Stolper E, Summons R, Treiman A, Williams R, Yingst A (2014) A habitable fluvio-

1036 lacustrine environment at Yellowknife Bay, Gale Crater, Mars. Science, 343.

1037 doi:10.1126/science. 1242777. 
1038 Gundlach B, Blum J (2013) A new method to determine the grain size of planetary

1039 regolith. Icarus, 223, 479-492. doi:/10.1016/j.icarus.2012.11.039

1040 Heavens NG, Kleinböhl A, Chaffin MS, Halekas JS, Kass DM, Hayne PO, McCleese

1041 DJ, Piqueux S, Shirley JH, Schofield JT, (2018) Hydrogen escape from Mars enhanced

1042 by deep convection in dust storms. Nature Astronomy, 2, 126-132. doi:10.1038/s41550-

$1043 \quad 017-0353-4$

1044 Hesselbrock AJ, Minton DA (2017) An ongoing satellite-ring cycle of Mars and the

1045 origins of Phobos and Deimos. Nature Geoscience, 10, 266-269. doi:10.1038/ngeo2916

1046 Hyodo R, Genda H, Charnoz S, Rosenblatt P (2017) On the impact origin of Phobos

1047 and Deimos. I. Thermodynamic and physical aspects. The Astrophysical Journal, 845,

1048 125. doi:10.3847/1538-4357/aa81c4

1049 Hyodo R, Kurosawa K, Genda H, Usui T, Fujita K (2019) Transport of impact ejecta

1050 from Mars to its moons as a means to reveal Martian history. Scientific reports, 9,

1051 19833. doi:10.1038/s41598-019-56139-x

1052 Hunten DM (1979) Capture of Phobos and Deimos by photoatmospheric drag. Icarus,

1053 37, 113-123. doi:10.1016/0019-1035(79)90119-2 
1054 Jacobson RA (2010) The orbits and masses of the Martian satellites and the libration of

1055 Phobos. The Astronomical Journal, 139, 668-679. doi:10.1088/0004-6256/139/2/668

1056 Jakosky BM, Pepin RO, Johnson RE, Fox JL (1994) Mars atmospheric loss and isotopic

1057 fractionation by solar-wind-induced sputtering and photochemical escape. Icarus, 111,

1058 271-288. doi:10.1006/icar.1994.1145

1059 Jakosky BM, Brain D, Chaffin M, Curry S, Grebowsky J, Grebowsky J, Halekas J,

1060 Leblanc F, Lillis R, Luhmann JG, Andersson L, Andre N, Andrews D, Baird D, Baker

1061 D, Bell J, Benna M, Bhattacharyya D, Bougher S, Bowers C, Chamberlin P, Chaufray

1062 J-Y, Clarke J, Collinson G, Combi M, Connerney J, Connour K, Correira J, Crabb K,

1063 Crary F, Cravens T, Crismani M, Delory G, Dewey R, DiBraccio G, Dong C, Dong Y,

1064 Dunn P, Egan H, Elrod M, England S, Eparvier F, Ergun R, Eriksson A, Esman T,

1065 Espley J, Evans S, Fallows K, Fang X., Fillingim M, Flynn C, Fogle A, Fowler C, Fox

1066 J, Fujimoto M, Garnier P, Girazian Z, Groeller H, Gruesbeck J, Hamil O, Hanley KG,

1067 Hara T, Harada Y, Hermann J, Holmberg M, Holsclaw, G, Houston S, Inui S, Jain S,

1068 Jolitz R, Kotova A, Kuroda T, Larson D, Lee Y, Lee C, Lefevre F, Lentz C, Lo D, Lugo

1069 R, Ma Y-J, Mahaffy P, Marquette ML, Matsumoto Y, Mayyasi M, Mazelle C, 
1070 McClintock W, McFadden J, Medvedev, A, Mendillo M, Meziane K, Milby Z, Mitchell

1071 D, Modolo R, Montmessin F, Nagy A, Nakagawa H, Narvaez C, Olsen K, Pawlowski

1072 D, Peterson W, Rahmati A, Roeten K, Romanelli N, Ruhunusiri S, Russell C, Sakai S,

1073 Schneider N, Seki K, Sharrar R, Shaver S, Siskind DE, Slipski M, Soobiah Y,

1074 Steckiewicz M, Stevens MH, Stewart I, Stiepen A, Stone S, Tenishev, V, Terada N,

1075 Terada K, Thiemann E, Tolson R, Toth G, Trovato J, Vogt M, Weber T, Withers P, Xu

1076 S, Yelle R, Yiğit E, Zurek R (2018) Loss of the Martian atmosphere to space: Present-

1077 day loss rates determined from MAVEN observations and integrated loss through time.

1078 Icarus, 315, 146-157. doi:10.1016/j.icarus.2018.05.030.

1079 Kallemeyn GW, Wasson JT (1981) The compositional classification of chondrites-I.

1080 The carbonaceous chondrite groups. Geochimica et Cosmochimica Acta, 45, 1217-

1081 1230. doi:10.1016/0016-7037(81)90145-9

1082 Kameda et al., Design of Telecopic Nadir Imager for Geomorphology (TENGOO) and

1083 Observation of surface Reflectance by Optical CHromatic Imager (OROCHI) for the

1084 MMX mission, This issue.

1085 Kawakatsu Y, Kuramoto K, Ogawa N, Ikeda H, Ono G, Sawada H, Imada T, Otsuki M, 
1086 Otake H, Muller R, Zacny K, Satoh Y, Yamada K, Mary S, Grebenstein M, Yoshikawa

1087 K (2019) Mission definition of Martian Moons eXploration (MMX), in 70th

1088 International Astronautical Congress. IAC-19, A3, 4B, 7, x51465.

1089 Kiuchi M, Nakamura AM (2014) Relationship between regolith particle size and

1090 porosity on small bodies. Icarus, 239, 291-293. doi:10.1016/j.icarus.2014.05.029

1091 Kleine T, Touboul M, Bourdon B, Nimmo F, Mezger K, Palme H, Jacobsen SB, Yin

1092 QZ, Halliday AN (2009) Hf-W chronology of the accretion and early evolution of

1093 asteroids and terrestrial planets. Geochimica et Cosmochimica Acta, 73, 5150-5188.

1094 doi:10.1016/j.gca.2008.11.047.

1095 Kobayashi M, Krüger H, Senshu H, Wada K, Okudaira O, Sasaki S, and Kimura H

1096 (2018) In situ observations of dust particles in Martian dust belts using a large-

1097 sensitive-area dust sensor, Planetary and Space Science, 156, 41-46.

1098 doi:10.1016/j.pss.2017.12.011

1099 Kokubo E, Ida S (1998) Oligarchic growth of protoplanets. Icarus, 131, 171-178.

1100 doi:10.1006/icar.1997.5840

1101 Lawrence DJ, Peplowski PN, Beck AW, Burks MT, Chabot NL, Cully MJ, Elphic RC, 
1102 Ernst CM, Fix S, Goldsten JO, Hoffer E M, Kusano H, Murchie SL, Schratz BC, Usui

1103 T, Yokley ZW (2019) Measuring the Elemental Composition of Phobos: The Mars-

1104 moon Exploration with GAmma rays and NEutrons (MEGANE) Investigation for the

1105 Martian Moons eXploration (MMX) Mission. Earth and Space Science, 6, 2605-2623.

1106 doi:10.1029/2019EA000811.

1107 Leshin LA, Mahaffy PR, Webster CR, Cabane M, Coll P, Conrad PG, Archer PD,

1108 Atreya SK, Brunner AE, Buch A, and Eigenbrode JL (2013) Volatile, isotope, and

1109 organic analysis of martian fines with the Mars Curiosity rover. Science, 341, 1238937.

1110 doi: $10.1126 /$ science. 1238937

1111 Mahaffy PR, Webster CR, Atreya SK, Franz H, Wong M, Conrad PG, Harpold D, Jones

1112 JJ, Leshin LA, Manning H, Owen T, Pepin RO, Squyres S, Trainer M, MSL Science

1113 Team (2013) Abundance and isotopic composition of gases in the Martian atmosphere

1114 from the Curiosity rover. Science, 341, 263-266. doi: 10.1126/science.1237966

1115 Michel P, Ballouz R-L, Barnouin OS, Jutzi M, Walsh KJ, May BH, Manzoni C,

1116 Richardson DC, Schwartz SR, Sugita S, Watanabe S, Miyamoto H, Hirabayashi M,

1117 Bottke WF, Connolly Jr., HC, Yoshikawa M, Lauretta DS (2020) Collisional formation 
1118 of top-shaped asteroids and implications for the origins of Ryugu and Bennu. Nature

1119 Communicationsn 11, 2665. doi:10.1038/s41467-020-16433-z

1120 Michel $\mathrm{P}$ et al. The MMX rover: roving and performing in-situ surface investigations on

1121 Phobos, This issue

1122 Mustard JF, Cooper CD, Rifkin MK (2001) Evidence for recent climate change on Mars

1123 from the identification of youthful near-surface ground ice. Nature, 412, 411-414.

1124 doi: $10.1038 / 35086515$

1125 Murray JB, Rothery DA, Thornhill GD, Muller JP, Iliffe JC, Day T, Cook AC (1994)

1126 The origin of Phobos' grooves and crater chains. Planetary and Space Sciences, 42, 519-

1127 526. doi:10.1016/0032-0633(94)90093-0

1128 Mumma MJ, Charnley SB (2011) The chemical composition of comets—emerging

1129 taxonomies and natal heritage. Annual Review of Astronomy and Astrophysics, 49,

1130 471-524. doi:10.1146/annurev-astro-081309-130811

1131 Nagao K, Okazaki R, Nakamura T, Miura YN, Osawa T, Bajo K, Matsuda S, Ebihara

1132 M, Ireland TR, Kitajima F, Naraoka H, Noguchi T, Tsuchiyama A, Yurimoto H,

1133 Zolensky ME, Uesugi M, Shirai K, Abe M, Yada T, Ishibashi Y, Fujimura A, Mukai T, 
1134 Ueno M, Okada T, Yoshikawa M, Kawaguchi J (2011) Irradiation history of Itokawa

1135 regolith material deduced from noble gases in the Hayabusa samples. Science, 333,

1136 1128-1131. doi:10.1126/science.1207785

1137 Nakamura T et al. Scientific observation plan of Phobos and Deimos from MMX

1138 spacecraft, This issue.

1139 Nishiizumi K, Arnold JR, Kohl CP, Caffee MW, Masarik J, Reedy RC (2009) Solar

1140 cosmic ray records in lunar rock 64455. Geochimica et Cosmochimica Acta, 73, 2163 -

1141 2176. doi:10.1016/j.gca.2008.12.021

1142 Pang KD, Rhoads JW, Lane AL, Ajello JM (1980) Spectral evidence for a

1143 carbonaceous chondrite surface composition on Deimos. Nature, 283, 277-278.

1144 doi:10.1038/283277a0

1145 Pang KD, Pollack JB, Veverka J, Lane AL, Ajello JM (1978) The composition of

1146 Phobos: Evidence for carbonaceous chondrite surface from spectral analysis. Science,

1147 199, 64-66. doi:10.1126/science.199.4324.64

1148 Pollack JB, Burns JA, Tauber ME (1979a) Gas drag in primordial circumplanetary

1149 envelopes: A mechanism for satellite capture. Icarus, 37, 587-611. doi:10.1016/0019- 
1151 Pollack JB, Colburn DS, Flasar FM, Kahn R, Carlston CE, Pidek D (1979b) Properties

1152 and effects of dust particles suspended in the Martian atmosphere. Journal of

1153 Geophysical Research: Solid Earth, 84, 2929-2945. doi:10.1029/JB084iB06p02929

1154 Poppe AR, Curry SM (2014) Martian planetary heavy ion sputtering of Phobos.

1155 Geophysical Research Letters, 41, 6335-6341. doi:10.1002/2014GL061100

1156 Ramsley KR, Head, JW (2013) Mars impact ejecta in the regolith of Phobos: Bulk

1157 concentration and distribution. Planetary and Space Science, 87, 115-129.

1158 doi:10.1016/j.pss.2013.09.005

1159 Rivkin AS, Brown RH, Trilling DE, Bell Iii JF, Plassmann JH (2002) Near-infrared

1160 spectrophotometry of Phobos and Deimos. Icarus, 156, 64-75.

1161 doi:10.1006/icar.2001.6767

1162 Rosenblatt P (2011) The origin of the Martian moons revisited. The Astronomy and

1163 Astrophysics Review, 19, 44. doi:10.1007/s00159-011-0044-6

1164 Rosenblatt P, Charnoz S, Dunseath KM, Terao-Dunseath M, Trinh A, Hyodo R, Genda

1165 H, Toupin S (2016) Accretion of Phobos and Deimos in an extended debris disc stirred 
1167 Senshu H et al. Development of LIDAR onboard MMX, This issue

1168 Soter S (1971) The dust belts of Mars. Report of Center for Radiophysics and Space

1169 Research, 462.

1170 Sugita S, Honda R, Morota T, Kameda S, Sawada H, Tatsumi E, Yamada M, Honda C,

1171 Yokota Y, Kouyama T, Sakatani N, Ogawa K, Suzuki H, Okada T, Namiki N, Tanaka

1172 S, Iijima Y, Yoshioka K, Hayakawa M, Cho Y, Matsuoka M, Hirata N, Hirata N,

1173 Miyamoto H, Domingue D, Hirabayashi M, Nakamura T, Hiroi T, Michikami T, Michel

1174 P, Ballouz R-L, Barnouin OS, Ernst CM, Schröder SE, Kikuchi H, Hemmi R, Komatsu

1175 G, Fukuhara T, Taguchi M, Arai T, Senshu H, Demura H, Ogawa Y, Shimaki Y,

1176 Sekiguchi T, Müller TG, Hagermann A, Mizuno T, Noda H, Matsumoto K, Yamada R,

1177 Ishihara Y, Ikeda H, Araki H, Yamamoto K, Abe S, Yoshida F, Higuchi A, Sasaki S,

1178 Oshigami S, Tsuruta S, Asari K, Tazawa S, Shizugami M, Kimura J, Otsubo T, Yabuta

1179 H, Hasegawa S, Ishiguro M, Tachibana S, Palmer E, Gaskell R, Le Corre L, Jaumann

1180 R, Otto K, Schmitz N, Abell PA, Barucci MA, Zolensky ME, Vilas F, Thuillet F,

1181 Sugimoto C, Takaki N, Suzuki Y, Kamiyoshihara H, Okada M, Nagata K, Fujimoto M, 
1182 Yoshikawa M, Yamamoto Y, Shirai K, Noguchi R, Ogawa N, Terui F, Kikuchi S,

1183 Yamaguchi T, Oki Y, Takao Y, Takeuchi H, Ono G, Mimasu Y, Yoshikawa K,

1184 Takahashi T, Takei Y, Fujii A, Hirose C, Nakazawa S, Hosoda S, Mori O, Shimada T,

1185 Soldini S, Iwata T, Abe M, Yano H, Tsukizaki R, Ozaki M, Nishiyama K, Saiki T,

1186 Watanabe S, Tsuda Y (2019) The geomorphology, color, and thermal properties of

1187 Ryugu: Implications for parent-body processes. Science, 364, eaaw0422.

1188 doi:10.1126/science.aaw0422

1189 Tolson RH, Duxbury TC, Born GH, Christensen EJ, Diehl RE, Farless D, Hildebrand

1190 CE, Mitchell RT, Molko PM, Morabito LA, Palluconi FD, Reichert RJ, Taraji H,

1191 Veverka J, Neugebauer G, Findlay JT (1978) Viking first encounter of Phobos:

1192 Preliminary results. Science, 199, 61-64. doi:10.1126/science.199.4324.61

1193 Thomas PC (1989) The shapes of small satellites. Icarus, 77, 248-274.

1194 doi:10.1016/0019-1035(89)90089-4

1195 Thomas PC (1993) Gravity, tides, and topography on small satellites and asteroids:

1196 Application to surface features of the Martian satellites. Icarus, 105, 326-344.

1197 doi:10.1006/icar.1993.1130 
1198 Thomas PC, Adinolfi D, Helfenstein P, Simonelli D, Veverka J (1996) The surface of

1199 Deimos: Contribution of materials and processes to its unique appearance. Icarus, 123,

1200 536-556. doi:10.1006/icar.1996.0177

1201 Usui T, Bajo KI., Fujiya W, Furukawa Y, Koike M, Miura YN, Sugawara H, Tachibana

1202 S, Takano Y, Kuramoto K (2020) The importance of Phobos sample return for

1203 understanding the Mars-moon system. Space Science Reviews, 216, 49.

1204 doi:10.1007/s11214-020-00668-9

1205 Villanueva GL, Mumma MJ, Novak RE, Käufl HU, Hartogh P, Encrenaz T, Tokunaga

1206 A, Khayat A, Smith MD (2015) Strong water isotopic anomalies in the martian

1207 atmosphere: Probing current and ancient reservoirs. Science, 348, 218-221.

1208 doi:10.1126/science.aaa3630

1209 Veverka J, Duxbury TC (1977) Viking observations of Phobos and Deimos:

1210 Preliminary results. Journal of Geophysical Research, 82, 4213-4223.

1211 doi:10.1029/JS082i028p04213

1212 Walsh KJ, Morbidelli A, Raymond SN, O'Brien DP, Mandell AM (2011) A low mass

1213 for Mars from Jupiter's early gas-driven migration. Nature, 475, 206-209. 
1215 Warren PH (2011) Stable-isotopic anomalies and the accretionary assemblage of the

1216 Earth and Mars: A subordinate role for carbonaceous chondrites. Earth and Planetary

1217 Science Letters, 311, 93-100. doi:10.1016/j.eps1.2011.08.047

1218 Wasson JT, Kallemeyn GW (1988) Compositions of chondrites. Philosophical

1219 Transactions of the Royal Society of London. Series A, Mathematical and Physical

1220 Sciences, 325, 535-544. doi:10.1098/rsta.1988.0066

1221 Watanabe S, Hirabayashi M, Hirata N, Hirata N, Noguchi R, Shimaki Y, Ikeda H,

1222 Tatsumi E, Yoshikawa M, Kikuchi S, Yabuta H, Nakamura T, Tachibana S, Ishihara Y,

1223 Morota T, Kitazato K, Sakatani N, Matsumoto K, Wada K, Senshu H, Honda C,

1224 Michikami T, Takeuchi H, Kouyama T, Honda R, Kameda S, Fuse T, Miyamoto H,

1225 Komatsu G, Sugita S, Okada T, Namiki N, Arakawa M, Ishiguro M, Abe M, Gaskell R,

1226 Palmer E, Barnouin OS, Michel P, French AS, McMahon JW, Scheeres DJ, Abell PA,

1227 Yamamoto Y, Tanaka S, Shirai K, Matsuoka M, Yamada M, Yokota Y, Suzuki H,

1228 Yoshioka K, Cho Y, Tanaka S, Nishikawa N, Sugiyama T, Kikuchi H, Hemmi R,

1229 Yamaguchi T, Ogawa N, Ono G, Mimasu Y, Yoshikawa K, Takahashi T, Takei Y, Fujii 
1230 A, Hirose C, Iwata T, Hayakawa M, Hosoda S, Mori O, Sawada H, Shimada T, Soldini

1231 S, Yano H, Tsukizaki R, Ozaki M, Iijima Y, Ogawa K, Fujimoto M, Ho T-M, Moussi

1232 A, Jaumann R, Bibring J-P, Krause C, Terui F, Saiki T, Nakazawa S, Tsuda Y (2019)

1233 Hayabusa2 arrives at the carbonaceous asteroid 162173 Ryugu-A spinning top-shaped

1234 rubble pile. Science, 364, 268-272. doi:10.1126/science.aav8032

1235 Yokota S, Saito Y, Asamura K, Tanaka T, Nishino MN, Tsunakawa H, Shibuya H,

1236 Matsushima M, Shimizu H, Takahashi F, Fujimoto M, Mukai T, Terasawa T (2009) First

1237 direct detection of ions originating from the Moon by MAP-PACE IMA onboard

1238 SELENE (KAGUYA). Geophysical Research Letters, 36. doi:10.1029/2009GL038185

1239 Yokota S, Terada N, Matsuoka A, Murata N, Saito Y, Delcourt D; Futaana Y, Seki K,

1240 Schaible MJ, Asamura K, Kasahara S, Nakagawa H, Nishino MN, Nomura R, Keika K,

1241 Harada Y, Imajo S, In situ observations of ions and magnetic field around Phobos: The

1242 Mass Spectrum Analyzer (MSA) for the Martian Moons eXploration (MMX) mission,

1243 This issue.

1244 Zellner BH, Capen RC (1974) Photometric properties of the Martian satellites. Icarus, 


\section{Figure titles and legends}

1248 Figure 1. The current design of spacecraft system configuration. (Top) From left to

1249 right, propulsion, exploration, and return modules. (Bottom) On-orbit configuration.

1250 The size of a solar panel is $2.4 \mathrm{~m} \times 4.4 \mathrm{~m}$. (C) JAXA

1251

1252 Figure 2. The mission profile of MMX. See text for details.

1253

1254 Figure 3. Planned observational orbits around Phobos. Quasi-satellites orbits (QSOs)

1255 are drawn in the Phobos fixed frame with Phobos at the center taking the xy-plane and

1256 the $\mathrm{x}$-axis direction to be the Phobos orbital plane and the opposite direction to Mars,

1257 respectively. Top: QSOs confined in the xy-plane. Bottom: 3D QSOs.

1258

1259 Figure 4. Outline of the tentative operation plan of MMX. The two panels are based on

1260 changes in the duration of eclipses by Mars and Phobos (top) and those in the

1261 configuration of Mars relative to the Sun and Earth (bottom), respectively. For eclipse

1262 duration estimates, a QSO-L orbit is assumed. CO: check out, H: QSO-H, M: QSO-M, 
1263 LA: QSO-LA, LB: QSO-LB, LC: QSO-LC, LSS: landing site selection, TD: touch

1264 down, DM: Deimos. Courtesy of Nakamura T and Ikeda H.

1265 
Table 1 Major properties of the Martian moons

\begin{tabular}{llll}
\hline Parameters & Phobos & Deimos & Ref. \\
\hline Mass $[\mathrm{kg}]^{1)}$ & $1.0626 \pm 0.0006 \times 10^{16}$ & $1.51 \pm 0.04 \times 10^{15}$ & 1 \\
Size $[\mathrm{km}]^{2)}$ & $13.3 \times 11.1 \times 9.1$ & $7.5 \times 6.1 \times 5.2$ & 2 \\
Volume $\left[\mathrm{km}^{3}\right]$ & $5621 \pm 154$ & $997 \pm 49$ & 2 \\
Mean density $\left[\mathrm{g} / \mathrm{cm}^{3}\right]$ & $1.89 \pm 0.05$ & $1.51 \pm 0.07$ & Mass/Vol. \\
Semi-major axis [Mars radius] & 2.76 & 6.92 & 3 \\
Eccentricity & 0.0151 & 0.00033 & 3 \\
Inclination $\left[{ }^{\circ}\right]^{3)}$ & 1.093 & 1.791 & 3 \\
Geometric albedo & $0.07 \pm 0.012$ & $0.068 \pm 0.007$ & 4,5 \\
\hline
\end{tabular}

1) $\mathrm{GM}$ values are converted to mass by adopting $\left.\mathrm{G}=6.67430 \times 10^{-11} \mathrm{~m}^{3} \mathrm{~kg}^{-1} \mathrm{~s}^{-2}, 2\right)$ semiaxes of the approximated triaxial ellipsoids, 3) relative to the Laplace plane

1. Jaconson (2020), 2. Thomas (1989), 3. JPL Solar System Dynamics, https://ssd.jpl.nasa.gov/, 4. Zellner and Capen (1974), 5. Thomas et al. (1996)

1269 Table 2. Observational pros (P) and cons (C) for hypotheses for the origin of the Martian

1270 Moons

\begin{tabular}{|c|c|c|c|c|}
\hline Evaluation item & \multicolumn{2}{|r|}{ Asteroid capture } & \multicolumn{2}{|r|}{ Giant impact } \\
\hline 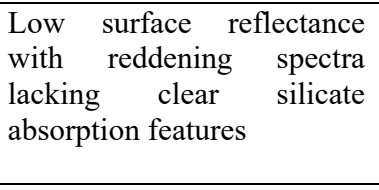 & $\mathrm{P}$ & $\begin{array}{l}\text { This resembles D-type asteroids, and so is } \\
\text { regarded as strong grounds for the asteroid } \\
\text { capture theory. }\end{array}$ & $\mathrm{N}$ & $\begin{array}{l}\text { No absorption feature indicative of ejected } \\
\text { Martian source materials is identified so far. } \\
\text { Note that this might be masked by strong } \\
\text { impact alteration or mixing of materials } \\
\text { from the impactor. }\end{array}$ \\
\hline $\begin{array}{l}\text { Small orbital eccentricity }(e) \\
\text { and inclination }(I) \text { relative to } \\
\text { the Martian equatorial plane }\end{array}$ & C & $\begin{array}{l}\text { It seems difficult, especially for Deimos, for } \\
\text { these orbits to evolve from a large } e \text { and } \\
\text { random } I \text { through tidal friction alone. }\end{array}$ & $P$ & $\begin{array}{l}\text { This is naturally explained if the giant } \\
\text { impact introduces most of the spin angular } \\
\text { momentum of Mars associated with the } \\
\text { generation of a Moon-forming debris disk. }\end{array}$ \\
\hline $\begin{array}{l}\text { Low-mass objects orbiting } \\
\text { within and beyond the co- } \\
\text { rotation radius }\left(r_{\mathrm{c}}\right) \text { with } \\
\text { Martian rotation }\end{array}$ & $\mathrm{N}$ & $\begin{array}{l}\text { Tidal evolution theory predicts Phobos } \\
\text { should be formed just inside } r \text { c, making the } \\
\text { conditions for capture severe. A similar } \\
\text { difficulty exists to explain Deimos's orbital } \\
\text { radius unless some frictional medium such } \\
\text { as a gas envelope extended to a large } \\
\text { distance. }\end{array}$ & $\mathrm{C}$ & $\begin{array}{l}\text { The formation of Deimos outside of } r_{\mathrm{c}} \\
\text { requires a large disk mass, resulting in the } \\
\text { formation of a massive inner moon. Tidal } \\
\text { friction could eventually lead such a moon to } \\
\text { falling onto Mars but geologic evidence for } \\
\text { such an impact is yet unidentified. }\end{array}$ \\
\hline
\end{tabular}


1274 (MO: mission objectives, MR: mission requirements, Priority: SS=very high, S=high, A=valuable)

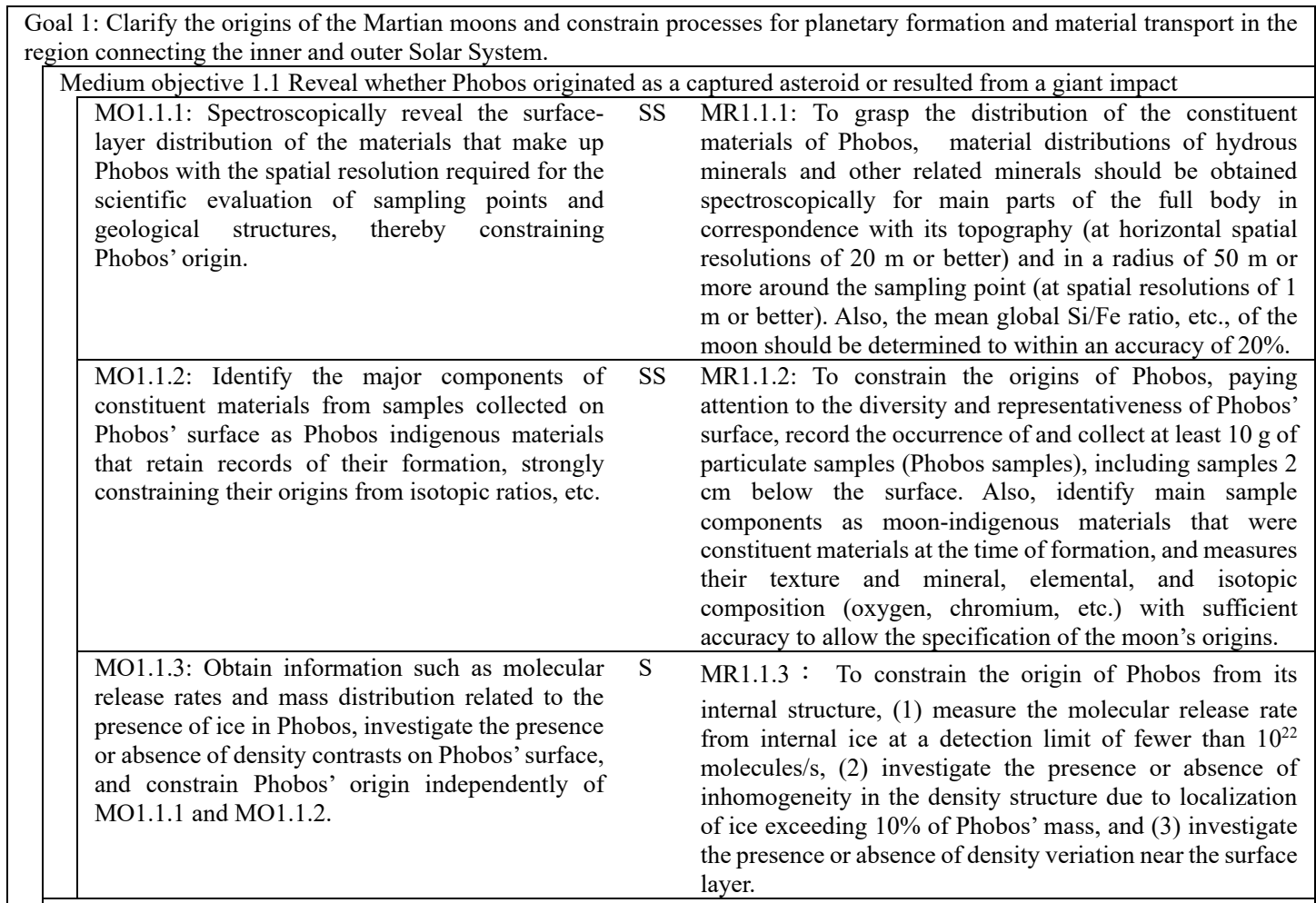

Medium objective 1.2a [If Phobos is determined to be a captured asteroid] Elucidate the composition and migration process of primitive materials supplied to the region of terrestrial planets and constrain the initial conditions of Martian surface evolution

MO1.2a.1: By constraining the formation of $\mathrm{S}$ MR1.2a: To constrain the initial evolution of Solar System primitive materials in the Solar System and primitive bodies in the vicinity of the snow line from a materials science perspective, and by estimating the Phobos capture process, constrain the initial conditions for the process of celestial body migration and evolution of the Martian surface in the early Solar System. materials and volatile element supply, analyze texture, element and isotope composition, formation age, etc., of moon-indigenous materials in Phobos samples with the necessary accuracy and also extract information related to organic matter and hydrous minerals. Also, elucidate collision environments before and after moon capture from the age distribution of impact metamorphisms in the collected sample and measurements of crater distributions on the moon surface.

Medium objective 1.2b [If Phobos is determined to originate from a giant impact] Elucidate giant impact and moon formation processes in the terrestrial planetary region and evaluate its influence on the early evolutionary process of Mars MO1.2b.1: For Phobos indigenous materials, S MR1.2b: To constrain the process of a giant impact, analyze identify primitive Martian components (Marsoriginating components) ejected by a giant impact and components of the impactor body, clarify their features, estimate the scale and age of the impact, and constrain celestial migration and planetary formation processes in the terrestrial planetary region. the texture, elemental and isotopic composition, metamorphic age, etc., of moon-indigenous materials in the Phobos sample with sufficient accuracy, and estimate the peak temperature, timing of the collision, and the mixing ratio of components from primitive Mars and the impacting body. Also, restrict the mixing ratio of both components across the entire moon from measurements of the $\mathrm{Si} / \mathrm{Fe}$ ratio, etc.

Medium objective 1.3 Place new constraints on Deimos' origin

MO1.3.1: Elucidate the surface distribution of $\mathrm{S}$ MR1.3: To grasp the distribution of constituent materials of materials composing Deimos through spectroscopy with the spatial resolution necessary for grasping its geological structures and compare this with Phobos. Deimos, from spectroscopic information, clarify the surface distribution of hydrous minerals and other related minerals corresponding to its topography at characteristic parts of the moon with a horizontal spatial resolution of $100 \mathrm{~m}$ or better.

Goal 2 From the viewpoint of the Martian moons, clarify the driving mechanism of the transition of the Mars-moon system, and add new knowledge to the evolution history of Mars.

Medium objective 2.1 Obtain a basic description of the elementary processes of surface evolution for moons in the circum-

Martian environment 


\begin{tabular}{|c|c|c|}
\hline $\begin{array}{l}\text { MO2.1.1: Identify weathering and evolutionary } \\
\text { processes (impact frequency, degree of gardening, } \\
\text { and space weathering processes) in surface-layer } \\
\text { regolith specific to the Martian moons as compared } \\
\text { to asteroids. }\end{array}$ & & $\begin{array}{l}\text { MR2.1: To know the surface evolution processes of Martian } \\
\text { moons, (1) observably constrain the circum-moons } \\
\text { environment, (2) grasp Phobos' geological features and } \\
\text { surface structures (craters, boulders, thickness and } \\
\text { deposition state of the regolith layer) at horizontal spatial } \\
\text { resolutions of } 20 \mathrm{~m} \text { or better, and (3) elucidate the state of } \\
\text { space weathering and metamorphism in Phobos samples. }\end{array}$ \\
\hline \multicolumn{3}{|c|}{ Medium objective 2.2 Add new findings and constraints on the history of changes in the Martian surface } \\
\hline $\begin{array}{l}\text { MO2.2.1: Search returned samples of the Phobos } \\
\text { surface for materials ejected from Mars after the } \\
\text { formation of Phobos and constrain the chemical } \\
\text { state of the Martian surface layer and its transition } \\
\text { if suitable samples are present. }\end{array}$ & A & $\begin{array}{l}\text { MR2.2.1: To constrain the chemical state and transition of } \\
\text { the surface layer of Mars, search a Phobos sample of } 10 \mathrm{~g} \\
\text { or more for materials (ejecta) from Mars from after moon } \\
\text { formation, and, if appropriate samples are present, clarify } \\
\text { features such as isotopic composition, formation age, and } \\
\text { remanent magnetization. }\end{array}$ \\
\hline $\begin{array}{l}\text { MO2.2.2: Place constraints on the amount of } \\
\text { atmospheric escape through the history of Mars } \\
\text { from composition ratios and isotopic ratios in the } \\
\text { current escaping atmosphere. }\end{array}$ & A & $\begin{array}{l}\text { MR2.2.2: To constrain the amount of atmospheric escape } \\
\text { through the history of Mars, measure composition ratios } \\
\text { and isotopic ratios of the main components of ions escaping } \\
\text { from the current Martian atmosphere to an accuracy within } \\
50 \% \text {. }\end{array}$ \\
\hline \multicolumn{3}{|c|}{$\begin{array}{l}\text { Medium objective } 2.3 \text { Constrain the mechanisms of material circulation in the Martian atmosphere affecting the transitions } \\
\text { in the Martian climate }\end{array}$} \\
\hline $\begin{array}{l}\text { MO2.3.1: Impose constraints on dust and water } \\
\text { transport processes in the Martian atmosphere and } \\
\text { between the atmosphere and the surface through } \\
\text { observations of the temporal changes in dust } \\
\text { storms and the global distributions of water vapor } \\
\text { and clouds. }\end{array}$ & $\mathrm{A}$ & $\begin{array}{l}\text { MR2.3: To constrain transport processes for dust and water } \\
\text { near the Martian surface, continuous observations of the } \\
\text { mid-to low-latitude distributions of dust storms, ice clouds, } \\
\text { and water vapor in the Martian atmosphere are performed } \\
\text { from high altitude equatorial orbit in different seasons to } \\
\text { within 1-hour time resolutions. }\end{array}$ \\
\hline
\end{tabular}


Table 4. Instruments onboard MMX for scientific observations

\begin{tabular}{|c|c|c|c|c|}
\hline \multirow{2}{*}{$\begin{array}{l}\text { Name: } \\
\text { function }\end{array}$} & \multirow{2}{*}{ Major specifications } & \multicolumn{3}{|c|}{ Roles } \\
\hline & & Moons' origin & Sampling assist & Mars system \\
\hline $\begin{array}{l}\text { LIDAR: }{ }^{1)} \\
\text { laser altimeter }\end{array}$ & $\begin{array}{l}\text { - Laser wavelength } 1064 \mathrm{~nm} \\
\text { - Ranging distance: } 100 \mathrm{~m}-100 \mathrm{~km} \\
\text { - Ranging resolution: }<22 \mathrm{~m} @ 100 \mathrm{~km} \\
\text { - Footprint } 50 \mathrm{~m} \text { from } 100 \mathrm{~km} \text { altitude } \\
\end{array}$ & \multirow{2}{*}{$\begin{array}{l}\text { Characterization of } \\
\text { geologic structures } \\
\text { and bedrock } \\
\text { exposed areas }\end{array}$} & \multirow{7}{*}{$\begin{array}{l}\text { Selection and } \\
\text { characterization of } \\
\text { sampling sites in } \\
\text { terms of safety for } \\
\text { landing, } \\
\text { geologic context, } \\
\text { and } \\
\text { representativeness } \\
\text { as pieces of bedrock }\end{array}$} & \multirow[t]{2}{*}{$\begin{array}{l}\text { Measurement of } \\
\text { surface } \\
\text { morphology for the } \\
\text { geologic history of } \\
\text { the moons }\end{array}$} \\
\hline $\begin{array}{l}\text { TENGOO: }{ }^{2)} \\
\text { telescopic } \\
\text { camera }\end{array}$ & $\begin{array}{l}\text { - FOV: } 1.1^{\circ} \times 0.82^{\circ} \\
\text { - Wavelength: a part of visible light } \\
\text { - Spatial resolution: } \sim 40 \mathrm{~cm} @ 20 \mathrm{~km} \text { alt. }\end{array}$ & & & \\
\hline $\begin{array}{l}\text { OROCHI: } \\
\text { wide-angle } \\
\text { multiband } \\
\text { camera }\end{array}$ & $\begin{array}{l}\text { - FOV: } 66^{\circ} \times 53^{\circ} \\
\text { - Wavelength (width): } 390(50), 480(30), 550(30) \text {, } \\
650(10), 700(10), 800(40), 950(60) \mathrm{nm} \text {, and } 400- \\
900 \mathrm{~nm} \text { (monochromatic) } \\
\text { - Spatial resolution: } 20 \mathrm{~m} @ 20 \mathrm{~km} \text { alt., } 10 \mathrm{~cm} \text { for an } \\
\text { area of } 100 \times 100 \mathrm{~m}^{2} \text { around landing sites, } 1 \mathrm{~mm} \text { for } \\
\text { an area of } 1 \times 1 \mathrm{~m}^{2} \text { around sampling sites }\end{array}$ & \multirow{4}{*}{$\begin{array}{l}\text { Determination of } \\
\text { global surface } \\
\text { composition } \\
\text { (Hydrous minerals, } \\
\mathrm{Fe} / \mathrm{Si}, \mathrm{H}_{2} \mathrm{O} \text { release } \\
\text { rate, etc.) }\end{array}$} & & \multirow[t]{2}{*}{$\begin{array}{l}\text { Observation of } \\
\mathrm{H}_{2} \mathrm{O} \text { and dust } \\
\text { circulation in the } \\
\text { Martian climate } \\
\text { system }\end{array}$} \\
\hline $\begin{array}{l}\text { MIRS:4) } \\
\text { near infrared } \\
\text { spectrometer }\end{array}$ & $\begin{array}{l}\text { - FOV: } 3.3^{\circ} \text { (in the direction of spectrometer slit) } \\
\text { - Wavelength: } 0.9-3.6 \mu \mathrm{m} \\
\text { - Spectral sampling: } 10 \mathrm{~nm} \\
\text { - Spatial resolution: }<20 \mathrm{~m} @ 20 \mathrm{~km} \text { alt. }\end{array}$ & & & \\
\hline $\begin{array}{l}\text { MSA: } \\
\text { ion mass } \\
\text { spectrometer }\end{array}$ & $\begin{array}{l}\text { - Geometric factor: } \geq 10^{4} \mathrm{~cm}^{2} \mathrm{sr} e V / e V \\
\text { - Ion energy: } \sim 5 \mathrm{eV} / \mathrm{q}-\sim 30 \mathrm{keV} / \mathrm{q} \text {, Energy resolution: } \\
\Delta \mathrm{E} / \mathrm{E} \sim 10 \% \\
\text { - Ion mass: } 1-100 \mathrm{amu}, \text { Mass resolution: } \mathrm{M} / \Delta \mathrm{M}>100 \\
\end{array}$ & & & $\begin{array}{l}\text { Searching for the } \\
\text { gas torus and } \\
\text { escaping Martian } \\
\text { atmosphere }\end{array}$ \\
\hline $\begin{array}{l}\text { MEGANE: } \\
\text { gamma-ray } \\
\text { and neutron } \\
\text { spectrometer }\end{array}$ & $\begin{array}{l}\text { - Gamma-ray energy: } 0.4-8 \mathrm{MeV} \text { with energy } \\
\text { resolution:<5.1 keV (FWHM) @ } 1454 \mathrm{keV} \\
\text { - Neutron energy: thermal }(0.01 \mathrm{eV}-0.5 \mathrm{eV}) \text {, } \\
\text { epithermal }(0.5 \mathrm{eV}-0.5 \mathrm{MeV}) \text {, fast }(0.5 \mathrm{MeV}-7 \\
\mathrm{MeV})\end{array}$ & & & \multirow[t]{2}{*}{ (not assigned) } \\
\hline $\begin{array}{l}\text { MMX Rover: } \\
\text { solar powered } \\
\text { rover }\end{array}$ & $\begin{array}{l}\text { - Laser Raman spectrometer } \\
\text { - Thermal radiometer } \\
\text { - Navigation cameras } \\
\text { - Wheel cameras } \\
\end{array}$ & $\begin{array}{l}\text { In situ measurement } \\
\text { of mineralogy and } \\
\text { physical properties } \\
\text { of regolith }\end{array}$ & & \\
\hline $\begin{array}{l}\text { CMDM: }{ }^{7)} \\
\text { dust counter }\end{array}$ & $\begin{array}{l}\text { - Dust size: }>10-20 \mu \mathrm{m} \\
\text { - Dust velocity: }>0.16 \mathrm{~km} / \mathrm{s}\end{array}$ & \multicolumn{3}{|c|}{$\begin{array}{l}\text { Detecting dust flux and ring to reveal the weathering process on the } \\
\text { moons' surface by micrometeoroid bombardments }\end{array}$} \\
\hline
\end{tabular}




\section{Figures}

Figure 1
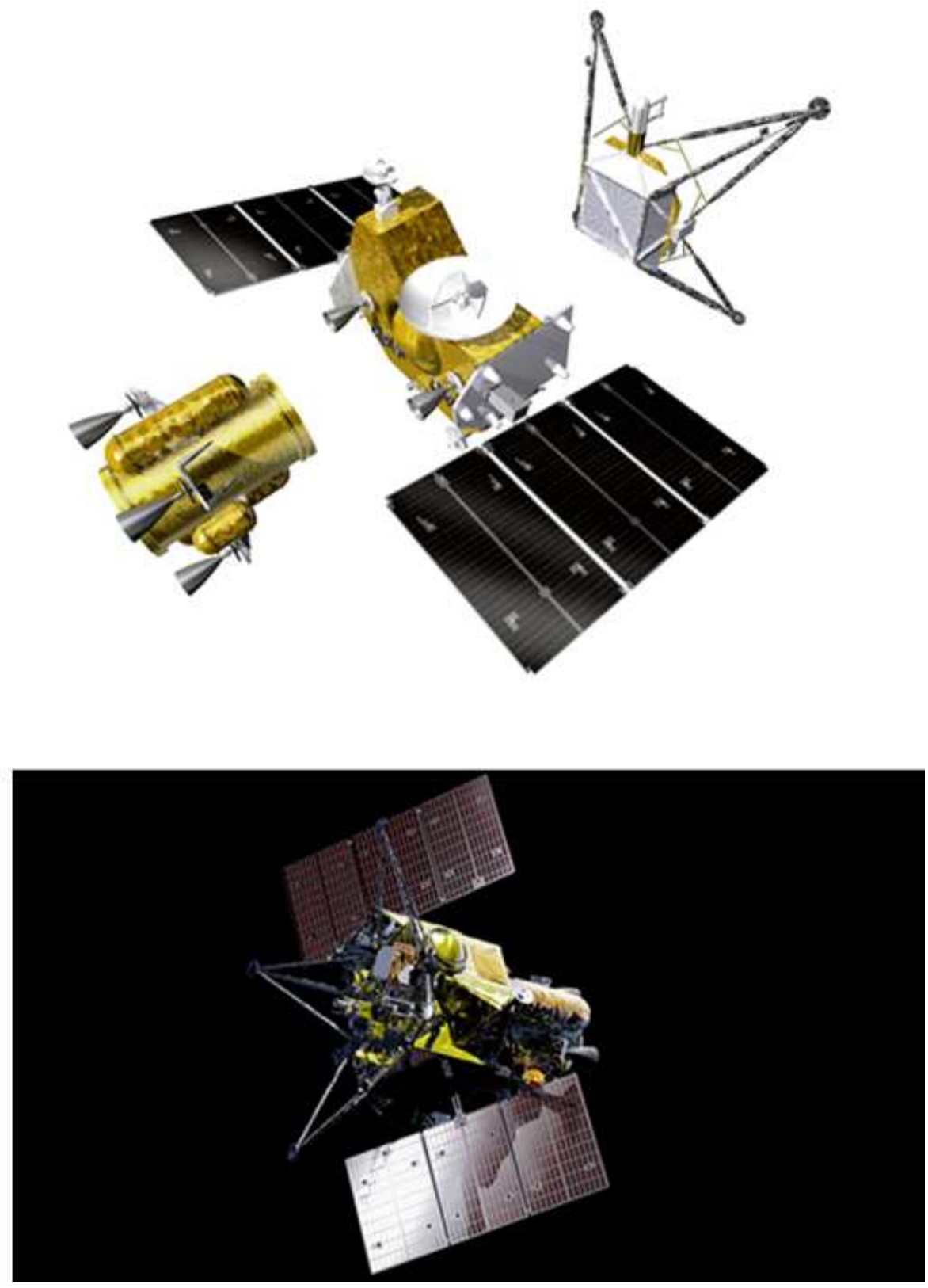

\section{Figure 1}

The current design of spacecraft system configuration. (Top) From left to right, propulsion, exploration, and return modules. (Bottom) On-orbit configuration. The size of a solar panel is $2.4 \mathrm{~m} \times 4.4 \mathrm{~m}$. (C) JAXA 
Figure 2

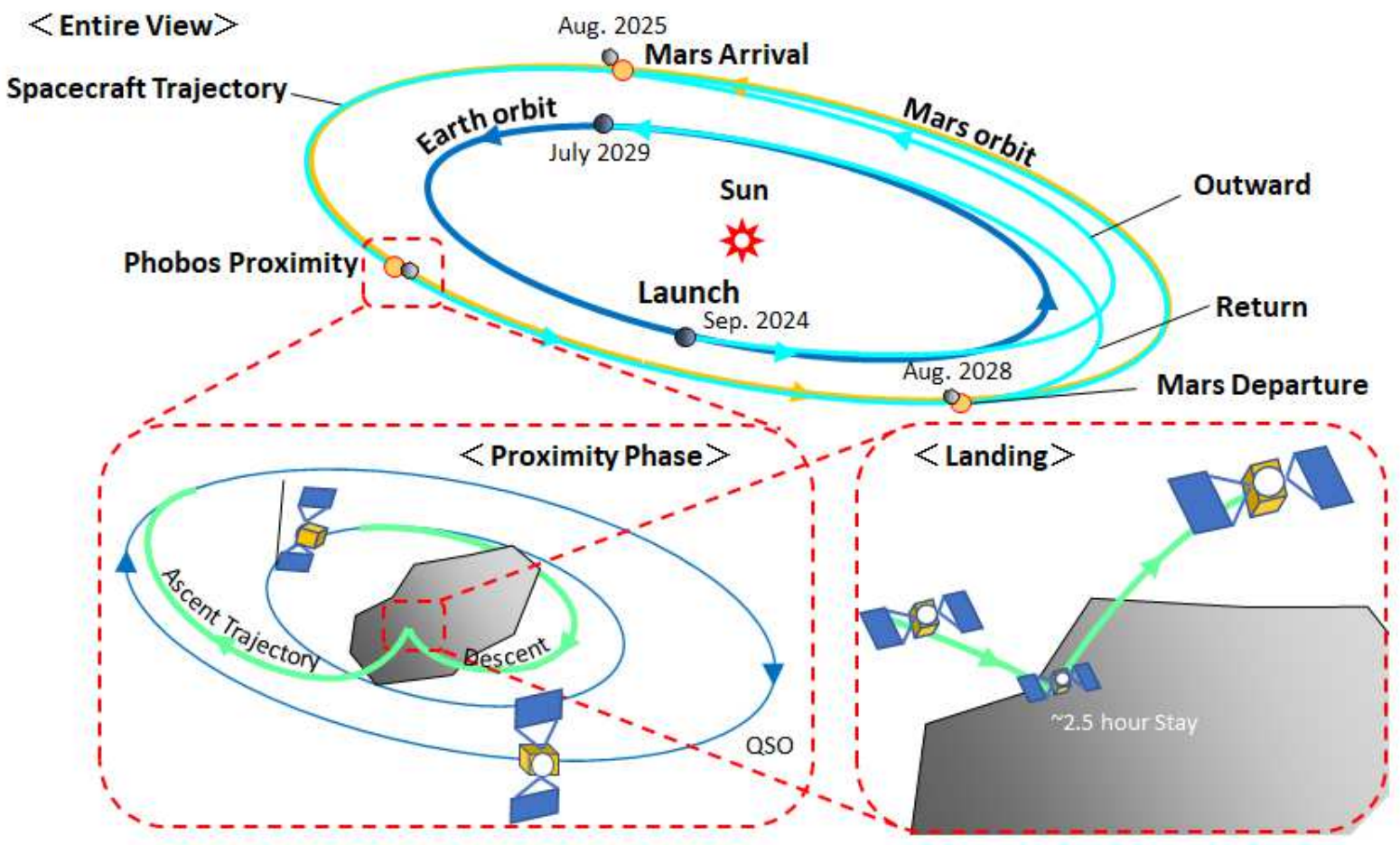

Figure 2

The mission profile of MMX. See text for details. 
Figure 3
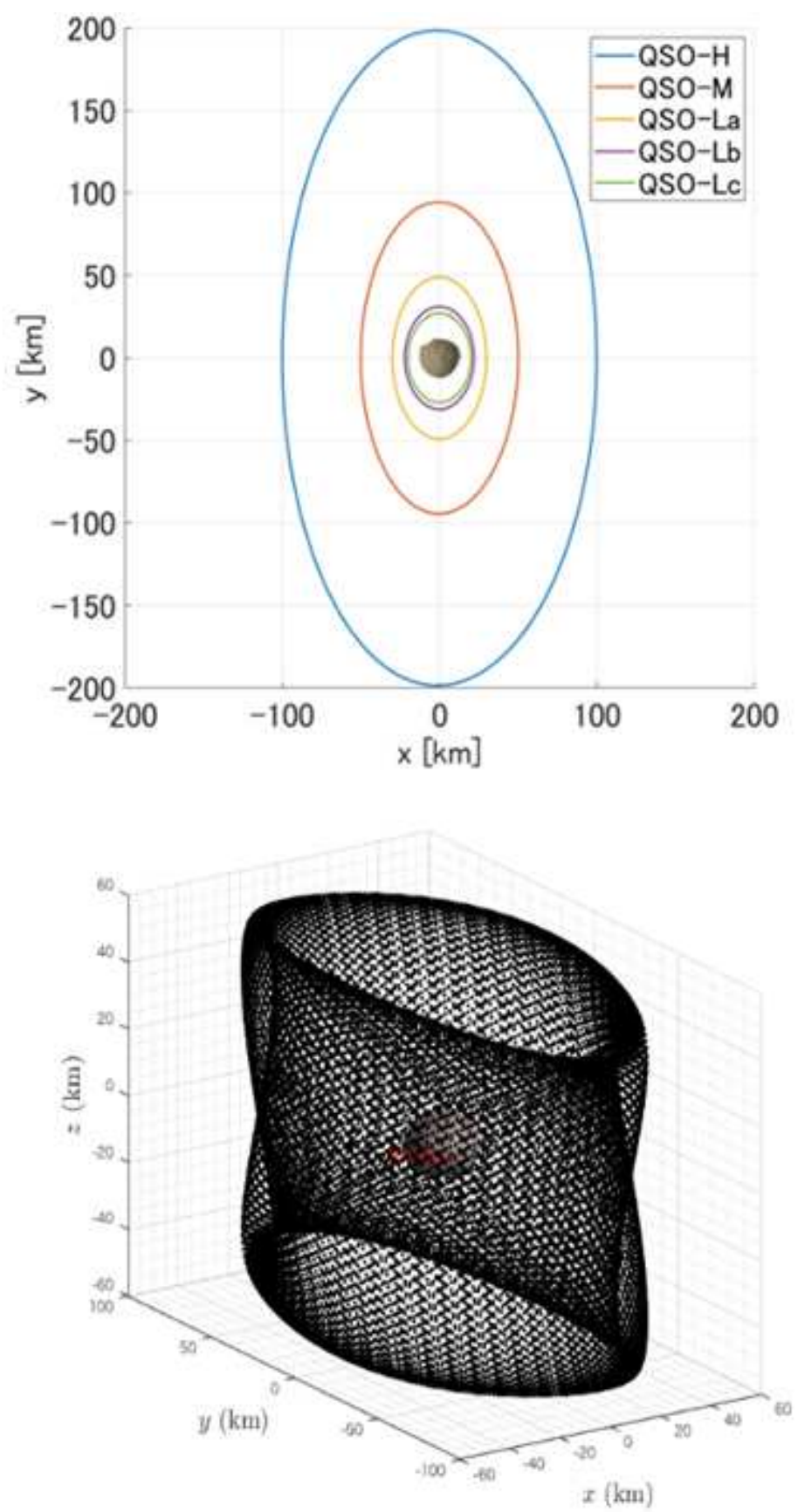

Figure 3

Planned observational orbits around Phobos. Quasi-satellites orbits (QSOs) are drawn in the Phobos fixed frame with Phobos at the center taking the xy-plane and the x-axis direction to be the Phobos orbital plane and the opposite direction to Mars, respectively. Top: QSOs confined in the xy-plane. Bottom: 3D QSOs. 


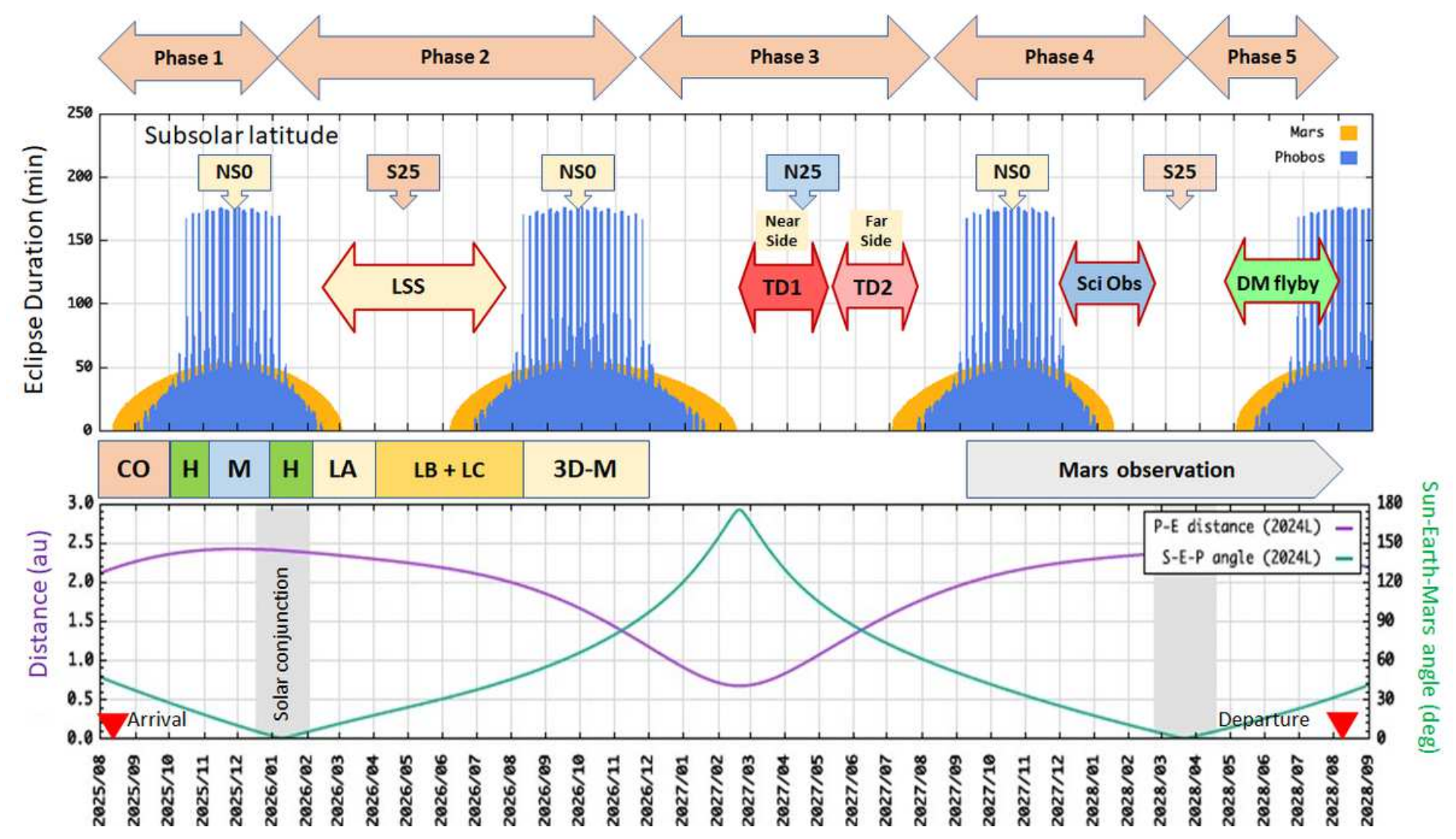

\section{Figure 4}

Outline of the tentative operation plan of MMX. The two panels are based on changes in the duration of eclipses by Mars and Phobos (top) and those in the configuration of Mars relative to the Sun and Earth (bottom), respectively. For eclipse duration estimates, a QSO-L orbit is assumed. CO: check out, H: QSO-H, M: QSO-M, LA: QSO-LA, LB: QSO-LB, LC: QSO-LC, LSS: landing site selection, TD: touch down, DM: Deimos. Courtesy of Nakamura $\mathrm{T}$ and Ikeda $\mathrm{H}$.

\section{Supplementary Files}

This is a list of supplementary files associated with this preprint. Click to download.

- graphicalabstract.png 